\title{
Title: Purification, characterization and influence on membrane properties of the plant-specific sphingolipids GIPC
}

\author{
Running title: Plant sphingolipids GIPCs
}

Adiilah Mamode Cassim¹, Yotam Navon², Yu Gao ${ }^{3}$, Marion Decossas ${ }^{4}$, Laetitia Fouillen $^{1}$, Axelle Grélard ${ }^{4}$, Minoru Nagano ${ }^{1,5}$, Olivier Lambert ${ }^{4}$, Delphine Bahammou ${ }^{1}$, Pierre Van Delft ${ }^{1}$, Lilly Maneta-Peyret ${ }^{1}$, Françoise Simon-Plas ${ }^{6}$, Laurent Heux ${ }^{2}$, Giovanna Fragneto ${ }^{7}$, Jenny C. Mortimer ${ }^{3}$, Magali Deleu ${ }^{8}$, Laurence Lins ${ }^{8}$, Sébastien Mongrand $^{1 *}$

1, Laboratoire de Biogènese Membranaire, UMR 5200, CNRS, Université de Bordeaux, 71 Avenue Edouard Bourlaux, 33883 Villenave d'Ornon Cedex, France

2, Centre de Recherches sur les Macromolécules Végétales (CERMAV), Univ. Grenoble Alpes, CNRS F-38000 Grenoble, France

3, Joint BioEnergy Institute, Emeryville, California 94608; Environmental and Systems Genomics, Lawrence Berkeley National Laboratory, Berkeley, California 94720

4, Institute of Chemistry \& Biology of Membranes \& Nanoobjects (UMR5248 CBMN), CNRS, Univ. Bordeaux, Institut Polytechnique Bordeaux, All. Geoffroy Saint-Hilaire, 33600 Pessac, France

5, College of Life Sciences, Ritsumeikan University, 1-1-1 Nojihigashi, Kusatsu, Japan

6, Agroécologie, AgroSup Dijon, CNRS, INRA, Univ. Bourgogne Franche-Comté, F-21000 Dijon, France

7, Institut Laue-Langevin ILL, 71 avenue des Martyrs, F-38042 Grenoble, France

8, Laboratoire de Biophysique Moléculaire aux Interfaces, TERRA Research Centre, GX ABT, Université de Liège, B-5030 Gembloux, Belgium

Present adress Adiilah Mamode Cassim : Agroécologie, AgroSup Dijon, CNRS, INRA, Univ. Bourgogne Franche-Comté, F-21000 Dijon, France

*Corresponding author: sebastien.mongrand@u-bordeaux.fr 


\section{Abstract}

The plant plasma membrane (PM) is an essential barrier between the cell and the external environment. The PM is crucial for signal perception and transmission. It consists of an asymmetrical lipid bilayer made up of three different lipid classes: sphingolipids, sterols and phospholipids. The most abundant sphingolipids in the plant PM are the Glycosyl Inositol Phosphoryl Ceramides (GIPCs), representing up to $40 \%$ of total sphingolipids, assumed to be almost exclusively in the outer leaflet of the PM. In this study, we investigated the structure of GIPCs and their role in membrane organization. Since GIPCs are not commercially available, we developed a protocol to extract and isolate GIPC-enriched fractions from eudicots (cauliflower and tobacco) and monocots (leek and rice). Lipidomic analysis confirmed the presence of different long chain bases and fatty acids. The glycan head groups of the different GIPC series from monocots and dicots were analysed by GC-MS showing different sugar moieties. Multiple biophysics tools namely Langmuir monolayer, $\zeta$-Potential, light scattering, neutron reflectivity, solid state ${ }^{2} \mathrm{H}-\mathrm{NMR}$ and molecular modelling were used to investigate the physical properties of the GIPCs, as well as their interaction with free and conjugated phytosterols. We showed that GIPCs increase the thickness and electronegativity of model membranes, interact differentially with the phytosterols species and regulate the gel-to-fluid phase transition during temperature variations.

\section{Keywords:}

Plant plasma membrane, sphingolipids, GIPC, biophysics, phytosterol, purification, lipidomics, glycolipid, molecular modelling, $\zeta$-Potential, Langmuir monolayer, neutron reflectivity, solid state NMR, cryo-EM

\section{Subject area:}

Plant plasma membrane organization, membrane domains, sphingolipid/sterol interaction, thermal homeoviscosity adaptation 
bioRxiv preprint doi: https://doi.org/10.1101/2020.10.01.313304; this version posted October 2, 2020. The copyright holder for this preprint (which was not certified by peer review) is the author/funder. All rights reserved. No reuse allowed without permission.

\section{Authors declare no conflict of interest}




\section{Introduction}

The plant plasma membrane (PM) contains three main classes of lipids: phytosterols, sphingolipids and phospholipids, all with a high level of molecular complexity, see (Cacas et al., 2016) (Yetukuri et al, 2008). In plants, the major sphingolipid subclass is the Glycosyl Inositol Phosphoryl Ceramides (GIPCs). GIPCs were discovered in plants and fungi during the 1950's (Carter et al., 1958). The structural diversity of GIPCs lies in the hydroxylation, degree and position of saturation of their fatty acid (FA) chain and long chain base (LCB) and glycosylation (Pata et al., 2010). Plant GIPCs predominantly consist of a t18:0 or t18:1 LCB (trihydroxylated saturated or monounsaturated) amidified to a Very Long Chain Fatty Acid (VLCFA) or 2-hydroxylated VLCFA (hVLCFA) to form a ceramide (Cacas et al., 2016)(Buré et al., 2011).

The GIPC head group linked to the ceramide consists of a phosphate bound to an inositol, forming the inositol phosphoryl ceramide (IPC) backbone, which is then further substituted with further sugar moieties. A broad study of the GIPC polar heads of 23 plant species from algae to monocots showed that polar head structures are largely unknown and vary widely across different biological taxa (Cacas et al., 2013). GIPCs are classified into series, based on the degree of glycosylation of their polar head group (Buré et al., 2011). In plants, all GIPCs characterized to date have a glucuronic acid (GlcA) as the first sugar on the IPC, followed by at least one more sugar unit of varying identity. For example, GIPC series $A$ is defined as one monosaccharide addition to the GlcA-IPC form (Buré et al., 2011). In the 1960s, the first characterization of a GIPC structure from Nicotiania tabacum (tobacco) was described (Carter et al., 1958)(Carter \& Kisic, 1969) (Carter \& Koob, 1969) (Hsieh, Lester, \& Laine, 1981) (Kaul \& Lester, 1978). The GIPC extraction method required hundreds of kilograms of plant material and litres of solvents. They fully resolved the exact number and type of sugars as well as the nature of the sugar bond. For instance, the reported series A GIPC still has the best described structure to date: $\operatorname{GlcNAc}(\alpha 1 \rightarrow 4) \operatorname{GlcA}(\alpha 1 \rightarrow 2)$ inositol-1-Ophosphorylceramide, see Figure $1 \mathrm{~A}$. Additional sugar moieties were described, such as glucosamine (GlcN), N-acetyl-glucosamine (GlcNAc), arabinose (Ara), galactose (Gal) and mannose (Man), which may lead to observed glycan patterns of three to seven 
sugars, the so-called GIPC series B to F. It is noteworthy that Kaul and Lester calculated the ratio between carbohydrate/LCB/inositol in purified polyglycosylated GIPCs and showed that they may contain up to 19-20 sugars (Kaul \& Lester, 1975), which opens a very large field of investigation. Polyglycosylated GIPCs found in Zea mays (corn) seeds and Erodium displays branched polar heads (Sperling \& Heinz, 2003)(Buré et al., 2016). GIPC series are species- and tissue- specific. In Arabidopsis, the GIPC series A headgroup Man-GlcA-IPC is predominant in leaves and callus (Mortimer et al., 2013) (Fang et al., 2016), whereas a complex array of $N$-acetylglycosylated with up to three pentose units are present in pollen (Luttgeharm et al., 2015). Amino- and N-acylated-GIPCs are found in Arabidopsis seeds and oil (Tellier et al., 2014). GlcN(Ac)-GlcA-IPC are mainly found in rice and tobacco (Buré et al., 2011) (Nagano et al., 2016). In monocots, the predominant GIPC series is series B (Buré et al., 2011), their core structures are yet to be deciphered.

The GIPC's polar head is responsible for the high polarity of the GIPC, accounting for its insolubility in traditional lipid extraction solvents, such as chloroform/methanol. Consequently, they are lost in the aqueous phase or at the interface. GIPCs, although one of the fundamental components of the plant PM model have been poorly studied, in part due to the absence of commercial preparations. Recent evidence has demonstrated that a loss of the glycosylation is lethal, (Ishikawa et al., 2018) (Rennie et al., 2014), and that misglycosylation affects both abiotic and biotic stress responses, as reviewed in (Mortimer \& Scheller, 2020).

Lipids are not homogeneously distributed within the PM bilayers. The lateral partitioning observed in the PM might be due to differential phase behaviours of different lipid species due to specific interactions between their different lipid species (Sampaio et al., 2009). This was reported in model membranes, using biophysical approaches and super resolution microscopy (Levental \& Veatch, 2016). Lipid domains or liquid-ordered (Lo) phases are formed from saturated phospholipids and sphingolipids in the presence of sterol, while liquid-disordered $(L d)$ phases are formed mainly from unsaturated phospholipids (Baumgart et al., 2007) (Lingwood \& Simons, 2010). In Lo phases, the high degree of conformational order is imposed on the acyl tails of lipids by the rigid ring 
structure of cholesterol. This increases the thickness of the lipid bilayer and lipid packing, although lipids remain laterally mobile (Mannock et al., 2010). Plant sterols and sphingolipid/sterol interactions have recently been reported as important determinants of lipid partitioning and organization within the PM (Beck et al., 2007; Gerbeau-Pissot et al., 2014; Grosjean et al., 2015). The plant PM contains 20-50\% sterols, depending on plant species and organ (Furt et al., 2007), harbouring a wide molecular diversity including free and conjugated species and dominated by $\beta$-sitosterol, stigmasterol, and campesterol (Moreau et al., 2018). These phytosterols play significant roles in regulating the order level of the membrane such that ternary mixtures (sterol/sphingolipid/saturated phospholipid) have less temperature sensitivity to thermal variations compared to systems mimicking the lipid rafts of animal and fungi (Beck et al., 2007). $\beta$-sitosterol and campesterol have the largest effect on lipid ordering (Beck et al., 2007; Cacas et al., 2016; Grosjean et al., 2015). Conjugated forms of $\beta$-sitosterol and stigmasterol are proposed to reinforce membrane cohesion by additional attractive van der Waals interactions with the acyl chains of sphingolipids and phospholipids (Beck et al., 2007). Using environment-sensitive probes, it was shown that various phytosterols have the ability to modulate the proportion of Lo phases and membrane heterogeneity in vivo as in vitro, with the notable exception of stigmaterol (GerbeauPissot et al., 2014) (Grosjean et al., 2015). Thus, GIPCs in synergy with sterols may organize and promote large ordered domains such that both have important roles in PM sub-compartmentalization and membrane dynamics (Grosjean et al., 2015).

As mentioned previously, older published protocols used large amounts of material and solvents, which is not feasible in modern labs. More recently published protocols do not yield enough material of high enough purity for structural characterization. In this project, we devised a new protocol to obtain milligram amounts of highly enriched GIPC samples from both monocots and eudicots, suitable for use in studies of GIPC structure and its role in PM organization. Using biophysics tools such as Langmuir monolayers, molecular modelling, supported lipid bilayers, giant unilamellar vesicles (GUVs), dynamic light scattering (DLS), ろ-potential, cryo-electron microscopy (cryo-EM), solid state ${ }^{2} \mathrm{H}-\mathrm{NMR}$ and neutron reflectivity, we aim to uncover the role of GIPCs, in synergy with sterols, in the plant PM organization. 
bioRxiv preprint doi: https://doi.org/10.1101/2020.10.01.313304; this version posted October 2, 2020. The copyright holder for this preprint (which was not certified by peer review) is the author/funder. All rights reserved. No reuse allowed without permission. 


\section{Results}

\section{Extraction and Purification of GIPC-enriched fractions from different plant species tissues and cell culture}

To assist with purifying the milligram amount of GIPCs required for analysis, we first assessed the amount of GIPCs in different plant species and tissues. We chose species/tissues which are easily and abundantly available, and quantified the nonhydroxylated VLCFA and 2-hydroxylated hVLCFA, diagnostic of plant's GIPC (Cacas et al., 2016). Four species were selected: 2 eudicot plants: cauliflower (Brassica oleacera, $\mathrm{Bo}$ ) head and Nicotiana tabacum (Nt) cell culture Bright-Yellow 2 (BY-2) and 2 monocot plants: the white leaves of leek (Allium porrum, $A p$ ) and rice (Oryza sativa, Oz) cell culture. The white part of plant tissues and cell cultures were used to avoid contamination by the abundant plastidial lipids and pigments. Cauliflower and rice cell culture have the highest GIPC content with an estimated $4.3 \mathrm{mg} / \mathrm{ml}$ and $3.4 \mathrm{mg} / \mathrm{ml}$ per fresh weight respectively (Figure 1B). BY2 cells and leek both had a much lower GIPC content, with a mean estimated content of $0.6 \mathrm{mg} / \mathrm{ml}$ and $0.4 \mathrm{mg} / \mathrm{ml}$ per fresh weight respectively.

GIPCs were extracted from all 4 materials to get different GIPC series. To maximize the yield, several trials were performed to test the different published protocols of GIPCs. Steps from three protocols were organized to obtain the best yield of GIPC. These protocols were from (Carter \& Koob, 1969), (Markham \& Jaworski, 2007) and (Kaul \& Lester, 1975). Figure 2 shows the extraction and purification processes to obtain GIPCenriched fractions of cauliflower (Bo-GIPC), tobacco BY-2 (Nt-GIPC), leek (Ap-GIPC) and rice (Os-GIPC). Some fine-tuning was done to maximize the yield such as refluxing in boiling ethanol for $20 \mathrm{~min}$ and using large lab-made silica column to process several hundreds of grams of material (see Material and Methods). Crude sphingolipid extracts were directly dried in silica deposited on the top of the column chromatography. The column was then washed with 4 column volumes $(\mathrm{cv})$ of a mix of chloroform/methanol with increasing polarity to remove sterols, glucosylceramide and phospholipids. For the elution of GIPCs, a step gradient of chloroform/methanol/water was used (Figure 2), so that molecules of increasing polarity were eluted in the last fractions. All washes and 
elution fractions were collected and analysed by high-performance thin layer chromatography (HPTLC) as shown in Supplementary data 1. HPTLC was a quick and reliable way to select fractions enriched with GIPCs, because it allowed the clear separation of sterols, phospholipids, and GIPC series.

\section{Fatty acid and sugar content of GIPC-enriched fractions}

To estimate the GIPC content, as well as the phospholipid contamination (medium chain fatty acid FA of C16-18), samples were trans-esterified in hot methanol/sulfuric acid solution to release both fatty acid-esterified glycerolipids and fatty acid-amidified sphingolipids. The samples were then derivatized by trimethyl-silylation, and analysed by Gas Chromatograph-Mass Spectrometry (GC-MS) using internal standards, which allowed quantification of total FA content. The percentage of fatty acid in the samples with medium chain length (\%FA) and hydroxylated and non-hydroxylated very long chain length (\%(h)VLCFA) were calculated from 2 to 3 independent experiments. Samples retained after step 1 (raw plant material), step 2 (crude sphingolipid extract) and step 3 (GIPC-enriched fractions) were analysed for their fatty acid content (Figure $3 A$ ). As we proceeded through the purification steps, the amount of medium chain FA decreased as the amount of (h)VLCFA increased (Figure 3A). At step 2, the percentage of (h)VLVCFA in the sphingolipid extract was around $50 \%$ and at the final step, the amount of (h)VLCFA was at about $80 \%$ for all GIPC-enriched extracts (Figure 3A). The detailed FA composition of the GIPC-enriched fractions of all four species is provided in supplementary data 2. It was estimated that the enrichment in GIPC between the first and last steps of the extraction and purification process was 5-fold for Bo-GIPC, 4.2-fold for Nt-GIPC, 3.6-fold for Ap-GIPC but only 2-fold for Os-GIPC.

The final products were analysed by HPTLC to verify the lipid composition, and they revealed the predominance of GIPCs (Figure 3B). Only traces of sterols and phospholipids were observed, and glucosylceramide (GluCer) was not detected. As reported in (Buré et al., 2011), eudicots contained mainly series $A$, monocots series $B$ and plant cells in liquid culture media a mix of GIPCs with highly glycosylated ones. The 
Bo-GIPC enriched fraction contained one major band of GIPC series A. The Nt-GIPC fraction contained GIPC series $A$ to $F$, further separated into three fractions ( $\alpha, \beta$ and $y$ ) of increasing polarity. The less polar fraction a contained two bands of series A GIPC closely packed together, representing PhytoSphingoLipid 1, PSL1 (with N-acetyl glucosamine) and PSL2 (with glucosamine) as described in (Kaul \& Lester, 1975) (Figure 3B), and a band of series $B$. The more polar fractions $\beta$ and $y$ showed the presence of the highly polyglycosylated D to F series GIPC (Figure 3B). As previously published monocots, Ap-GIPC and Os-GIPC as enriched fractions contained mainly GIPC series B, and with some series $A$ and polyglycosylated GIPCs also present (Figure 3B).

The predominant GIPC-derived (h)VLCFA species was dependent on the starting material. The Bo-GIPC enriched fraction consisted of h24, h24:1 and h26 as the main fatty acyl chain, Nt-GIPC with h24, h22, h23 and h25 acyl chain, Ap-GIPC with C24, h24, h22 and C22 and Os-GIPC with C24, C22, C20 and h24 (Figure 4A).

We next investigated the sugar moieties present in GIPC-enriched fractions by high performance anion exchange chromatography (HPAEC) coupled with Pulsed Amperometric Detection (PAD), a technique used to detect underivatized monosaccharide sugars (Figure 4B). Since different glycosidic bonds hydrolyse at different rates, and some sugars rings are more sensitive to cleavage than others, the sugar content of the Bo-GIPC enriched fraction was analysed $1 \mathrm{~h}, 3 \mathrm{~h}$ and $4 \mathrm{~h}$ after trifluoroacetic acid (TFA) hydrolysis. Results showed that hydrolysis has no to very little effect on the sugar moieties of GIPC fractions (Supplementary data 3). As expected, all GIPC-enriched fractions contained glucuronic acid (GlcA) found in GIPC samples previously characterized, see for review (Mamode Cassim et al., 2019). The Bo-GIPC enriched fraction not only contains glucose (Glc) and mannose (Man) previously found in Brassicaceae species (Fang et al., 2016), but also large amount of arabinose (Ara) and galactose (Gal). These latter sugars, never described in Brassicaceae such as Arabidopsis, could be a real specificity of Bo-GIPC's polar head, or due to cell wall/glycoprotein contamination of the GIPC-enriched fraction, see further below. 
The different fractions of the Nt-GIPC series have a complex glycan content. Fraction a contained glucuronic acid (GlcA), glucosamine (GlcN) and mannose (Man) (Figure 4B). Note here that $\mathrm{N}$-acetyl glucosamine (GlcNAc) is hydrolysed during the extraction procedure and is mixed with GlcN. Gal and Ara became the main glycan moieties in fractions $\beta$ and $y$ as described for highly glycosylated GIPC, series D (Kaul \& Lester, 1978). Monocot GIPC-enriched sample, both Ap-GIPC and Os-GIPC contained mainly Man, Gal and GlcN at relatively equal amount, and GlcA and Ara at lower amount (Figure 4B).

Previous studies have suggested interactions between GIPCs and cell wall components, particularly the pectin Rhamnogalacturonan II (RGII) (Voxeur \& Fry, 2014). However, we did not detect either galacturonic acid (GalA) nor rhamnose (Rha), two main components of pectins, suggesting no major pectin contamination (Figure 4B). We detected, however, a large amount of Ara and Gal (Figure 4B). A Yariv reactivity test (Kitazawa et al., 2013) was performed to check for the presence the arabino-galactan (AG) as contaminants in the GIPC-enriched fractions (Figure $4 \mathrm{C}$ ). No zone of clearance was observed, suggesting no detectable AG in each GIPC sample (50 $\mu \mathrm{g})$. Gum arabic and saline buffer were used as positive and negative controls respectively (Figure 4C). The potential contamination of the GIPC samples by proteins was also tested using the Bradford method. However, in GIPC samples of up to $30 \mu \mathrm{g}$, no protein was detected (data not shown).

The Bo-GIPC and Ap-GIPC purified fractions were analysed by LC-MS and compared to total sphingolipids extracted from crude cauliflower or crude leek. Results showed in Supplementary data 4 revealed an absence of CER and gluCER contamination in the purified GIPC sample, and that the LCB and FA content is very similar except a slight loss of h24:0/1- and t18: 0/1-containing GIPC (less that 10\%), see Supplementary data 5.

\section{Biophysical characterisation of the GIPC-sterol interaction}


We decided to focus on the Bo-GIPC to perform various biophysical analyses. We first characterized the lipid-lipid interactions at the micrometric level by the Langmuir trough compression technique applied on a monolayer model at the air-water interface (Deleu et al., 2014). As previously reported (Cacas et al., 2016), experimental biophysical characterization coupled to energetic calculations suggested a preferential interaction of GIPC series A with $\beta$-sitosterol, defined as a 'condensing effect', with the favourable interaction minimizing the energy of interaction, i.e. where the interfacial area occupied by the 2 molecules is less than the interfacial area occupied by single one. To further characterize the interaction of Bo-GIPC with phytosterols, we conducted biophysical experiments to investigate the outer leaflet organization with free and conjugated sterols ( $\beta$-sitosteryl Glucoside, SG and Acyl (18:2) $\beta$-sitosteryl Glucoside, ASG). The ratio of GIPC:sterol (80:20 mol ratio) is consistent with the estimated ratio of the lipids in the outer leaflet of the PM (Cacas et al., 2016)(Tjellstrom et al., 2010). The compression isotherm of Bo-GIPC (green line) (Figure 5A) shows a low and relatively constant surface pressure in large molecular areas, corresponding to a 'gaseous' state. Compression of the monolayer induced a progressive increase in surface pressure, indicating the appearance of a liquid-expanded state (in agreement with the twodimensional compressibility modulus, $\mathrm{Cs}^{-1}$, of $38.3 \mathrm{mN} \mathrm{m}^{-1}$ in the 160 - to $110-\AA^{2}$ per molecular region), which is characterized by a certain degree of condensing interaction between the molecules at the interface (Figure 5A). The mean interfacial area of BoGIPC is $212.9 \pm 4.9 \AA^{2}$ in its expanded form and at its most condensed form is $60.0 \pm$ 14.6 $\AA^{2}$. These results are in agreement with the results previously obtained with Nicotiana tabaccum-GIPCs (Cacas et al., 2016).

The interaction of Bo-GIPC mixed with different sterols was assessed by the thermodynamic analysis of the compression isotherms of mixed GIPC-sterol monolayers. In this comparative study, we adhere to the rule of additivity, which suggests that if two molecules within a mixed monolayer are immiscible, the area occupied by the mixed film will be the sum of the areas of the separated components. The deviation to that rule is attributed to the existence of specific interaction between the two molecules (Maget-Dana, 1999). The mean molecular area of the mixed monolayer Bo-GIPC: $\beta$-sitosterol $(80: 20)$ was lower than the calculated theoretical value 
(using the rule of additivity), at the estimated physiological membrane surface pressure of $30 \mathrm{mN} \mathrm{m}^{-1}$ (Marsh, 1996) (Figure 5B). This condensing effect of $\beta$-sitosterol in presence of Bo-GIPC confirms that previously reported for tobacco GIPCs (Cacas et al., 2016). The trend was however reversed for the mixed monolayers of Bo-GIPC:SG $(80: 20)$ and Bo-GIPC:stigmasterol (80:20), where the mean molecular area is significantly higher than the theoretical value (Figure 5B). For Bo-GIPC:ASG (80:20), the effect is intermediate. The most significant difference between the experimental and theoretical mean molecular area was obtained for the mixed monolayer BoGIPC:stigmasterol (80:20). Interestingly, the only structural difference between $\beta$ sitosterol and stigmasterol is the presence of a double bond at C22 in stigmasterol. The mixed monolayer GIPC:ASG (80:20) had a comparable mean molecular area to GIPC molecule at low surface area (Figure 5A) and the average difference between the mean molecular area and its theoretical value is $30 \AA^{2}$ per molecule for all three surface pressures (Figure 5B).

In order to thermodynamically analyse the interaction of the two components and the stability of the mixed monolayer, the excess free energy of the mixing ( $\Delta \mathrm{Gex})$ and the free energy of mixing $(\Delta \mathrm{GM})$ were respectively calculated for all four mixed monolayers (Figure $5 \mathrm{C}$ ). The negative value of $\Delta \mathrm{Gex}$ for the mixed monolayer GIPC: $\beta$-sitosterol (80:20) suggested a strong attractive interaction between the two components and the negative value of $\triangle \mathrm{GM}$ indicated thermodynamic stability of the mixed monolayer (Figure 5C) as suggested by (Cacas et al., 2016). The values of $\Delta \mathrm{Gex}$ and of $\Delta \mathrm{GM}$ for the mixed monolayers GIPC:SG (80:20), GIPC:ASG (80:20) and GIPC/Stigmasterol (80:20) were both positive in all three mixed monolayers (Figure 5C) showing repulsion between the molecules within the monolayer and thermodynamic instability of the mixed monolayers.

\section{Modelling the interaction between GIPC and phytosterols}

Hypermatrix is a simple docking method used to calculate specific interactions between two amphiphilic molecules (for a review see (Deleu et al., 2014). The interaction of one molecule of GIPC series $A$ with t18:0/h24:0 and one molecule of sterol was generated in silico using this method and analysed. The sterols used were 
the four molecules studied by the Langmuir monolayer technique i.e. $\beta$-sitosterol, stigmasterol, ASG, and SG (Figure 6). The interacting molecules displayed very different configurations. The differences between the spatial organization of the GIPC/sitosterol and GIPC/stigmasterol were striking: the a-side of the steryl moities of $\beta$-sitosterol was directed towards the acyl chains of the GIPC, whereas the steryl rings of stigmasterol was positioned at a perpendicular angle with respect to GIPC hydrocarbon chains (Figure 6A,B). In mammals the interaction of the a face of cholesterol with lipid acyl chains favours its condensing effect (Rog \& Pasenkiewiczgierula, 2004). This was notably established by comparing the effects of cholesterol and lanosterol, which possesses a methyl group on the a face, on lipid organization (Yeagle et al., 1977)(Smondyrev \& Berkowitz, 2001)(Róg et al., 2009). For stigmasterol, the structural difference of the unsaturation on $\mathrm{C} 22$ seems thus to modify its interaction with GIPC (Figure 6B), and this can be correlated to the non-condensing effect observed experimentally in the monolayer compression experiments (Figure 5). Similarly, the $\beta$ face of the steryl ring moiety of the steryl glucoside (SG) was oriented towards GIPC acyl chains. It is noteworthy that the bending of the sugar head group of GIPC favours its interaction with the glucose head group of SG (Figure 6C). In the conformation of the conjugated sterol ASG, the acyl chain is in direct interaction with the a side of the sterol, such that the $\beta$-side of the steryl cycle interacts with GIPC acyl chains (Figure 6B). Thus, $\beta$-sitosterol is the only sterols tested for which the interaction of its a-face with the GIPC acyl chains is favoured. This could be related to its condensing effects observed experimentally.

\section{Effect of GIPC on membrane organization and thickness}

To further investigate the properties of GIPC using model bilayers, we tried to make large unilamellar vesicles (LUV) with sizes between 50-500 nm with Bo-GIPC by freeze/thawing. We found that GIPC alone made aggregates but not vesicles (Figure 7A). Fluorescent microscopy observations of Nt-GIPC containing LUV in water at RT, $\mathrm{pH} 7$ led to similar results (Supplementary data 6A). However, by adding phospholipids we could observe the formation of LUV (Supplementary data 5A). To closer mimic the outer leaflet of the PM enriched in GIPCs, we generated LUV with a ternary system of 
GIPC:phospholipid: $\beta$-sitosterol (1:1:1). As phospholipids, we used 1-palmitoyl-2linoleoyl-sn-glycero-3-phosphocholine (PLPC), 1-palmitoyl-2-oleoyl-sn-glycero-3phosphocholine (POPC) or dioleoyl-sn-glycero-3-phosphocholine (DOPC), in all cases the ternary system yielded liposomes using the freeze-thaw method with liquid nitrogen and water bath of $60^{\circ} \mathrm{C}$ (Figure 7A) (Supplementary data 6B). Giant unilamellar vesicles (GUVs) were also made using the Teflon method by (Kubsch et al., 2017) with a ternary mix of GIPC/DOPC/sitosterol (Figure 7B). The incorporation of GIPCs into the liposomes were analysed by dynamic light scattering (DLS) which gives the hydrodynamic diameter of the liposomes. The addition of GIPC did not seem to affect the hydrodynamic diameter of liposomes, which was about $100 \mathrm{~nm}$. The $\zeta$-potential of the GIPC-containing liposomes was measured to be around $-26 \mathrm{mV}$, while DOPC/ $\beta$ sitosterol alone had a $\zeta$-potential of $-5 \mathrm{mV}$ (Figure $7 \mathrm{C}$ ). The difference in $\zeta$-potential between GIPC and GIPC-free liposomes is attributed to the fact that GIPCs are negatively charged because of the presence of the glucuronic acid, and furthermore, confirms that GIPC was indeed incorporated into the lipid membrane. (Jiang et al., 2019) showed that the $\zeta$-potential of the surface of wild-type Arabidopsis mesophyll cells are at $-20 \mathrm{mV}$ which is quite close to that of our liposomes. It seems that GIPC contributes significantly in the negative potential of the plant PM outer leaflet, which might influence, for example, its interaction with cell wall components. The structure of the LUVs made by the freeze/thaw method was further investigated by cryo-EM. GIPC/POPC-- ${ }^{2} \mathrm{H}_{31} / \quad \beta$-sitosterol- and GIPC/POPC- ${ }^{2} \mathrm{H}_{31} /$ stigmasterol-containing LUVs formed not only regular-shaped bilayer vesicles, but also planar bilayer structures that seem more rigid and not able to bend and make proper vesicles (arrow in Figure 8). Comparison of the bilayer thickness of these GIPC-containing LUVs with LUVs containing only POPC and sterols, showed a significant difference of thickness from 4.5 $\mathrm{nm}$ for the ternary LUV to 3.5 for the binary LUV (Figure 8).

We also investigated the influence of GIPCs on membrane thickness by neutron reflectivity (Figure 9A). Supported Lipid Bilayers (SLB) were formed by vesicle fusion of liposomes containing POPC, GIPC and constant $\beta$-sitosterol concentration. Three different membrane compositions were tested with increasing (0, 15 and $30 \% \mathrm{~mol}$ ) GIPC concentration. GIPC. The reflectivity profile was analysed, and following model 
fitting, the scattering length density profile and the thickness of the polar head and acyl tail in the bilayer were obtained. The results showed that liposomes containing $30 \%$ mol. of GIPC did not form a continuous bilayer on the surface, as indicated by a high solvent content in the hydrophobic tail region. This implies that the high GIPC content modified the bilayer properties, such that it did not adhere to the support. However, 0 and $15 \%$ mol containing GIPC liposomes did form continuous bilayers. The addition of GIPCs increased the bilayer thickness by $8 \AA$, as compared to GIPC-free SLBs, due to the $4 \AA$ of sugar head group in each layer (Figure $9 \mathrm{~A}$ ). Refer to the tables of Figure 9B for more details of the structural parameters that were generated. Figure $9 \mathrm{C}$ shows a scattering length density profile of the SLBs.

\section{Phase transition of GIPC containing-vesicles analysed by liquid state ${ }^{2} \mathrm{H}-\mathrm{NMR}$}

Finally, using solid-state ${ }^{2} \mathrm{H}-\mathrm{NMR}$, we asked whether GIPCs have an effect on the gelto-fluid phase transition of a fully hydrated binary and ternary lipid mix system. Solidstate ${ }^{2} \mathrm{H}$-NMR spectroscopy is a non-intrusive method giving structural and dynamic information about lipid bilayers (Davis, 1983; Seelig, 1977). Here, we aimed to find the nature of the membrane phases, their dynamics, and how GIPCs and phytosterols are regulating the membrane phase transition, such as the well-described effect of cholesterol on the membrane (Oldfield et al., 1978).

We used Bo-GIPC enriched fractions to make membrane systems using deuterated palmitoyl-oleoyl phosphatidylcholine containing 31 atoms of deuterium on the palmitoyl chain $\left(\right.$ POPC- ${ }^{2} \mathrm{H}_{31}$ ) as a probe for solid-state ${ }^{2} \mathrm{H}-\mathrm{NMR}$. We chose to use POPC as it is a phospholipid with a long chain fatty acid with an unsaturation found in plant PM (Cacas et al., 2016) and a gel-to-fluid transition temperature of $-2.5^{\circ} \mathrm{C} \pm 2.4$ (Koynova \& Caffrey, 1998). We generated liposomes using the freeze/thaw method as previously described. Figure 10A showed ${ }^{2} \mathrm{H}$ NMR spectra of two lipid mix systems containing GIPC (GIPC/ POPC- ${ }^{2} \mathrm{H}_{31} / \beta$-sitosterol (1:1:1, mol ratio) and GIPC/ POPC- ${ }^{2} \mathrm{H}_{31} /$ stigmasterol (1:1:1, mol ratio) and two control samples without GIPC (POPC- ${ }^{2} \mathrm{H}_{31} / \beta$-sitosterol $(1: 1, \mathrm{~mol}$ ratio) and POPC- ${ }^{2} \mathrm{H}_{31} /$ stigmasterol $(1: 1, \mathrm{~mol}$ ratio). Spectra were acquired by varying the temperature from $-10^{\circ} \mathrm{C}$ to $40^{\circ} \mathrm{C}$. These are plausible thermal variations that plants may experience in nature. The obtained ${ }^{2} \mathrm{H}$ NMR spectra exhibit the typical powder pattern 
line shape with a spectral width decreasing as the temperatures increase. This qualitative observation can be supplemented by a quantitative characterization using the first spectral moment (Davis, 1983). Figure 10B shows the temperature plots of first moments (M1) calculated from ${ }^{2} \mathrm{H}-\mathrm{NMR}$ powder spectra of liposomes with or without BoGIPCs, as well as pure POPC- ${ }^{2} \mathrm{H}_{31}$. On figure 10B left, we can hence appreciate the phase transition of a pure POPC- ${ }^{2} \mathrm{H}_{31}$ membrane such that the low M1 corresponds to the fluid $(L d)$ phase and the high $M 1$ to the rigid $(L o)$ phase. The thermal variation showed an abolished phase transition upon adding phytosterols to $\mathrm{POPC}-{ }^{2} \mathrm{H}_{31}$ (Figure $10 \mathrm{~B}$ left). This abolition is more pronounced for $\beta$-sitosterol with a higher ordering effect above the phase transition temperature compared to stigmasterol. These conclusions can be transposed to ternary systems with the difference that $\beta$-sitosterol has a stiffening effect at low temperatures (Figure 10B right). Above the POPC- ${ }^{2} \mathrm{H}_{31}$ phase transition both GIPC and phytosterol were able to rigidify the membrane, with a larger effect for $\beta$-sitosterol. This result is similar to those obtained by Beck et al, 2007. Taken together, these experiments showed that GIPC and phytosterols adopt the same behaviour as cholesterol, and hence have a high propensity to regulate fluidity during temperature variations. 


\section{Discussion}

\section{Fine-tuning GIPC purification}

GIPCs are quantitatively and qualitatively essential components of plant plasma membranes (Cacas et al., 2013; Mortimer et al., 2013). As there are no commercially available GIPCs, reasonable quantities of these molecules must be isolated with good purity to study their biophysical properties. This paper describes a protocol for efficient purification of milligram amount of GIPCs. It has been inspired by three publications (Carter \& Koob, 1969),(Kaul \& Lester, 1975)(Markham \& Jaworski, 2007), whereby the steps were rearranged in order to get rid of contamination such as sterols and glycerolipids during the extraction procedure, while retaining GIPCs which were eluted in the column purification steps. This is simpler and more convenient than the one used in previous publications where large amounts of solvents were used. It is also timeefficient and achieve a reasonable yield since we were able to obtain $30 \mathrm{mg}$ of GIPC of up to $85 \%$ purity from 4 distinct plant materials of $600-800 \mathrm{~g}$ fresh weight. In the near future, to improve yield and purity, we still have to fine-tune the purification and extraction process of GIPCs, and it will also be important to purify different GIPC series individually, so as to decipher the number, bonds and types of sugar residues which make up the different plant GIPC polar head groups, as was done for fungi GIPC by NMR (Simenel et al., 2008) (Gutierrez et al., 2007). To do so, ad hoc preparative chromatography needs to be developed with elution solvents with the right polarity.

The full structure and diversity of sugar moieties in the GIPCs polar head remains to be understood and investigated. The diversity seems to be important, for example in plant/pathogen interactions. A recent study showed that the GIPC polar head may be receptor for oomycete necrotic toxins called Necrosis and ethylene-inducing peptide 1like (NLPS). Plants enriched in GIPC series A are sensitive to NLPs while those enriched in GIPC series B are insensitive to NLPs, hence conferring resistance against pathogens secreting NLPs (Lenarčič et al., 2017). 
The polar head sugar diversity of GIPCs are clearly species-dependent (Buré et al., 2011). For instance, the increasingly large amounts of Gal and Ara in the Nt-GIPC fractions has been described in (Kaul \& Lester, 1978) where GIPCs of tobacco leaves contain up to 4 Ara and 2 Gals attached to the core GIPC structures of GlcN/GlcNAcGlcA-Ins-P-Cer. Hence, it is correct to assume that the large amount of Ara and Gal of Nt-GIPC(fraction y) derives from the polyglycosylated GIPCs of up to GIPC series $E$ of Ara-Ara-Gal-Gal-Man-GlcN/GIcNAc-GlcA-Ins-P-Cer.

In all enriched GIPC fractions purified in this study, GalA and Rha are absent (Figure $4 \mathrm{~B})$, suggesting no contamination by pectins, particularly RGIl, which is reported to bind to GIPCs (Voxeur \& Fry, 2014). It should be noted that Ara and Gal can form AG side chains of the pectin Rhamnogalacturonan I (RGI). Ara and Gal are also the dominant sugars in the abundant GPI-anchored plasma membrane glycoproteins, the arabinogalactan proteins (AGPs). To test for the presence of RGI or AGPs, we performed a Yariv reactivity test, but we did not detect anything that could bind to Yariv.

Previous work in Arabidopsis thaliana (At, also a Brassicaceae, like cauliflower), identified a mannose as the first sugar attached to the GlcA-Ins-P-Cer core in vegetative tissues, a reaction catalysed by the glycosyltransferase AtGMT1 (Fang et al., 2016). We hypothesized that this also might be the case in cauliflower. In the seed tissue of $A t$, Glucosamine InositolPhosphorylCeramide Transferase 1 (AtGINT1), another glycosyltransferase, adds a GlcNAc instead of a Man to the core structure of GIPC (Ishikawa et al., 2018). However, in both cases, the authors also detected Ara and Gal as part of the sugar composition of their GIPC enrichments. Therefore, we cannot yet conclude whether Ara and Gal are contaminations or are inherent sugar moieties of GIPC.

\section{GIPCs alone form aggregates, but not liposomes}

With the aim of studying the physical properties of GIPC, we attempted to make liposomes (LUV). Since GIPCs have large polar heads and VLCFAs, they tend to agglomerate, similarly to pure sterols. In order to make liposomes and further study GIPCs, a binary mix with phospholipids, or a ternary mix with phospholipids and sterols 
were used. To be closer to the biological PM model, we used a lipid mix of GIPC/phospholipid/sterol at a molar ratio of 1:1:1. As expected for lipids with (h)VLCFAs, GIPCs increase the thickness of the model membrane by a few $\mathrm{nm}$, as shown by neutron reflectivity on a supported bilayer. The bilayer thickness of liposomes containing GIPCs as observed using cryo-EM are around 6-7 nm for the ternary mix, which corresponds well with the observed thickness of purified PM from Medicago truncatula and tobacco (Lefebvre et al., 2007)(Mongrand et al., 2004).

One important feature of the PM is its electrostatic charge. PM purification using polymers phase separation PEG/Dextran relies on the fact that PMs are highly negatively charged and that the PM right-side-out ( $\mathrm{RSO}$ ) fraction is attracted to the positively charged PEG phase (Morré \& Morre, 2000). The membrane surface charge (MSC) is regulated by lipids and post transcriptional modification of proteins such as phosphorylation (Goldenberg \& Steinberg, 2010). The $\zeta$-potential of GIPC-containing liposomes is $-26 \mathrm{mV}$, five times higher than a PC/ $\beta$-sitosterol -containing bilayer, likely due to the large negativity of GIPC conferred by its phosphate group and the GlcA residue of the polar head. Therefore, since GIPCs are mostly located in the outer leaflet of the PM (Cacas et al., 2016), we conclude here that GIPCs contribute strongly to the negative charge of the PM outer leaflet.

\section{GIPC interaction with sterols and effects on the membrane order}

Using the biophysical techniques of Langmuir compression isotherms and molecular modelling, we showed that GIPCs interact differentially with different phytosterols. We confirmed that GIPCs with $\beta$-sitosterol has a condensation effect as described in (Cacas et al., 2016) whereas non condensing interactions occur between stigmasterol, SG or ASG and GIPCs (Figures 5, 10). These differential interactions appear to be structure dependent. Just adding a glucose head group (SG) and an acyl chain (ASG) or an unsaturation (C22 in stigmasterol) to the $\beta$-sitosterol steryl moieties changes the interaction with the GIPCs and modifies the properties of the model membranes (Figure 5, 6 and 7). Interestingly, GIPCs, ASG and SG all accumulate after drought stress (Tarazona et al., 2015). The differential interaction of GIPCs with the different kind of sterols could explain how plants cope with such stress. 
As mentioned, stigmasterol also displayed a non-condensation effect. The structural difference between sitosterol and stigmasterol is only an unsaturation on C22. This has a dramatic effect on membrane fluidity as discussed in (Grosjean et al., 2015). Using model membranes and environment-sensitive probes, they showed that plant lipids promote various spatial organization of membrane and that $\beta$-sitosterol promotes Lo phases while stigmasterol has a low ordering effect and is correlated with low level of Lo phases. Plant sterols and sphingolipids form lipid rafts which are signalling platforms (Mongrand et al., 2004)(Gronnier et al., 2018). These structures can be clearly seen as lipid domains in model monolayers containing $\beta$-sitosterol and GIPC that interact with each other (Figure 7). This interaction might translate into Lo phases. Stigmasterol, on the other hand, tends to sequester small structures containing GIPC which might contribute to membrane fluidity.

Plants are poikilothermic and have to adapt the viscosity of their membrane to temperature changes, a process called homeoviscosity. By modulating the fluidity of their membrane to be functionally viable, plants can adapt to temperature fluctuations. For example, plants can readily convert $\beta$-sitosterol to stigmasterol by expressing the C22 desaturase CYP710 during temperature acclimation (Morikawa et al., 2006). Specific plant membrane components like $\beta$-sitosterol, stigmasterol and glucosylcerebrosides are synthesized as part of temperature adaptations to make membrane-associated biological processes possible (Beck et al., 2007). Here, we showed that GIPCs are more conducive to enable homeoviscosity. It will be interesting to further investigate how GIPCs are involved in modulating PM fluidity in thermal adaptation in synergy with other PM lipid components.

\section{GIPC structure in membrane organization}

Recent studies provide new insight on the importance role of GIPC structure in plants through genetic approach (Fang et al., 2016; Ishikawa et al., 2018; Jiang et al., 2019; Mortimer et al., 2013). By generating mutants combined to the multidisciplinary approaches, we can uncover more about GIPC intricate structure and its biological 
implications. The modification of the ceramide length and hydroxylation of GIPC might alter the organization of the membrane as does sphingomyelin (SM) in animal cell, which is responsible for interdigitating between the bilayers and domain formation with cholesterol (Róg et al., 2016) (London \& Brown, 2000). The closest biological molecule in terms of membrane structuring role of plant GIPC series $A$ and $B$ could be SM, even if the latter -absent in plant PM- is made up of a phosphocholine head group. The theoretical model of plant PM showed GIPC as the major sphingolipid in the outer leaflet, just like sphingomyelin, and inducing a lateral segregation to form Lo phases with phytosterols (Cacas et al., 2016)(Tjellstrom et al., 2010). Interestingly, the exact distribution of sterols in the two layers of the PM is still a matter of debate, including in animal research fields (Courtney et al., 2018). To know where sitosterol or stigmasterol are located and how they regulate the fluidity of one or both of the PM leaflets is of great interest. Unfortunately, the tools to study phytosterol distribution remain to be developed.

Plant GIPCs are structurally homologous to the animal gangliosides that are absent in plants. Gangliosides are acidic glycolipids containing sialic acid in their polar head that play an important role in immunity, signal transduction in the PM that essential for brain and retinal functions in animal cells (Sonnino \& Prinetti, 2010)(Sibille et al., 2016). It is possible that polyglycosylated GIPCs have a similar role to gangliosides. Further investigation will require a better understanding of the GIPC glycosylation pattern, and the enzymes involved in GIPC biosynthesis. The present study paves the way for tackling the function of plant glycosylated sphingolipids in membrane organisation and function. 


\section{Material and Method}

\section{Plant Material}

Cauliflower and leek were store-bought. Wild-type tobacco (cv. Bright Yellow) cell culture and rice cell culture were obtained as previously described in (Cacas et al., 2013). and (Nagano et al., 2016) respectively.

\section{Extraction and purification of GIPCs}

The green parts of the cauliflower and leek were removed to prevent contamination by galactolipids, which are mainly present in chloroplasts. Plant material $(800 \mathrm{~g}$ fresh weight) was blended with 5 litres of cold $0.1 \mathrm{~N}$ aqueous acetic acid in a chilled stainlesssteel Waring Blender at low, medium and high speed for 30s each. The slurry was filtered through 16 layers of acid-washed Miracloth. The residue was re-extracted once (twice for leek) again in the same manner. The aqueous acetic acid filtrate was discarded. The residue was air-dried overnight under a fume hood and was then refluxed with 2 litres of hot $70 \%$ ethanol $(0.1 \mathrm{~N}$ in $\mathrm{HCl})$ for $20 \mathrm{~min}$. The slurry was filtered hot through 16 layers of Miracloth pre-washed with acidic ethanol (pressed well to remove all liquid). This process was repeated twice more using a total of 5 litres of acidic ethanol. The combined filtrates were chilled at $-20^{\circ} \mathrm{C}$ for $48 \mathrm{~h}$. The precipitate was removed by centrifugation at $30,000 \mathrm{~g}$ ( $14000 \mathrm{rpm}$ at using a Sorvall SLA-1500 rotor) at $4^{\circ} \mathrm{C}$ for $15 \mathrm{~min}$. Sphingolipids were then extracted from the precipitates in hot isopropanol/hexane/water $(55: 20: 25, \mathrm{v} / \mathrm{v})$. The solution was homogenized using an Ultra-Turrax for $20 \mathrm{~s}$ and incubated at $60^{\circ} \mathrm{C}$ for $20 \mathrm{~min}$. After centrifugation at $3000 \mathrm{~g}$ for $10 \mathrm{~min}$, the supernatant was decanted to another tube and the residue extracted twice more with the hot solvent. A total of $100 \mathrm{ml}$ of solvent was used at this step. The supernatants were combined and its lipid content was analysed by TLC and GC-MS to evaluate the amount of GIPC content.

Porous silica beads (Silica gel for chromatography $60 \AA$, 75-125 um, Acros Organics), were used throughout for packing the column chromatography. The column consists of $70 \mathrm{ml}$ of silica beads, sand of Fontainebleau, followed by the sphingolipid sample dried in $20 \mathrm{ml}$ of silica beads (see Figure 2). The column was washed and equilibrated with chloroform. Apolar lipids were washed with a mix of chloroform/methanol of different volume ratios of increasing polarity (4:1 then $3: 1$ and 2:1). The volume used was equivalent to 4 -fold the volume of the column. The column was then eluted with a step gradient of chloroform:methanol:water. Solvent A was chloroform:methanol:water (59:37.5:3.5, v/v) and the solvent B chloroform:methanol:water $(46: 42: 12, \mathrm{v} / \mathrm{v})$. The step gradient elution started with $100 \%$ A to end with $100 \% \mathrm{~B}$, with $10 \%$ intervals. The volume of elution corresponds to 2 -fold the volume of the column. $1 / 100^{\text {th }}$ of each elution fractions were collected and dried for GC-MS and TLC analysis to test the purity 
of the fractions. Fractions containing the same type of GIPCs were pooled and dried. The estimated quantity of GIPC is assessed by calculating the amount of (h)VLCFA ((hydroxylated-) Very Long Chain Fatty Acid). (h)VLCFA represents $1 / 3$ of total GIPC molecular mass.

\section{High performance thin-layer chromatography analysis}

High-performance thin-layer chromatography (HP-TLC) plates were Silicagel 60 F254 (Merck, Rahway, NJ). HP-TLC plates were impregnated for 3 min with freshly prepared $0.2 \mathrm{M}$ ammonium acetate in methanol, and further dried at $110 \mathrm{C}$ for $15 \mathrm{~min}$. Purified lipids as well as crude extracts were chromatographed in Chloroform/Methanol/ 4N $\mathrm{NH}_{4} \mathrm{OH}(9: 7: 2, v / v)$ on. Lipids were located under UV after staining with Primuline in acetone/water $80 / 20$.

\section{Carbohydrate Analysis}

Samples $(0.2 \mathrm{mg})$ were hydrolysed with fresh $2 \mathrm{M} \mathrm{TFA}$ at $120^{\circ} \mathrm{C}$ for either $1 \mathrm{~h}, 3 \mathrm{~h}$, or 4 $\mathrm{h}$. The supernatants were retained, dried in a vacuum concentrator, redissolved in $2 \mathrm{~mL}$ of water and filtered through $0.22 \mu \mathrm{m}$ filters. Samples were analysed by HPAEC on an ICS-5000 instrument (Thermo Fisher Scientific) equipped with a CarboPac PA20 analytical anion exchange column ( $3 \mathrm{~mm} \times 150 \mathrm{~mm}$; Thermo Fisher Scientific), a PA20 guard column ( $3 \mathrm{~mm} \times 30 \mathrm{~mm}$; Thermo Fisher Scientific), a borate trap, and a pulsed amperometric detector. The column was equilibrated with $40 \mathrm{mM} \mathrm{NaOH}$ for 5 min before injection of the sample. Monosaccharides were separated using the following methods: a linear gradient from $4 \mathrm{mM} \mathrm{NaOH}$ to $3 \mathrm{mM} \mathrm{NaOH}$ in the first $6 \mathrm{~min}$, followed by a linear gradient of $3 \mathrm{mM} \mathrm{NaOH}$ to $1 \mathrm{mM} \mathrm{NaOH}$ from 6 to $8 \mathrm{~min}$. An isocratic gradient was held at $1 \mathrm{mM} \mathrm{NaOH}$ from 8 to $23 \mathrm{~min}$, and then increased to $450 \mathrm{mM} \mathrm{NaOH}$ to elute the acidic sugars from $23.1 \mathrm{~min}$ to $45 \mathrm{~min}$. Monosaccharide standards were used for quantification.

\section{Fatty Acid Analysis}

Each sample was transmethylated at $110^{\circ} \mathrm{C}$ overnight in methanol containing $5 \%(\mathrm{v} / \mathrm{v})$ sulfuric acid and spiked with $10 \mathrm{mg}$ of heptadecanoic acid (c17:0) and $10 \mathrm{mg}$ of 2hydroxy-tetradecanoic acid (h14:0) as internal standards. After cooling, $3 \mathrm{~mL}$ of $\mathrm{NaCl}$ $(2.5 \%, \mathrm{w} / \mathrm{v})$ was added, and the released fatty acyl chains were extracted in hexane. Extracts were washed with $3 \mathrm{~mL}$ of saline solution $(200 \mathrm{mM} \mathrm{NaCl}$ and $200 \mathrm{mM}$ Tris, $\mathrm{pH}$ 8), dried under a gentle stream of nitrogen, and dissolved in $150 \mathrm{~mL}$ of BSTFA and trimethylchlorosilane. Free hydroxyl groups were derivatized at $110^{\circ} \mathrm{C}$ for $30 \mathrm{~min}$, surplus BSTFA-trimethylchlorosilane was evaporated under nitrogen, and samples were dissolved in hexane for analysis using GC-MS under the same conditions as described (Buré et al., 2011). Quantification of fatty acids and hydroxyl acids was based on peak 
areas, which were derived from total ion current, and using the respective internal standards.

\section{Langmuir monolayer trough}

Purified GIPC-enriched fractions were used in this study. A solution at $0.4 \mathrm{mM}$ in chloroform:methanol:water (30:60:8) was prepared. Sterols and PLPC were purchased from Avanti Polar Lipids. They were dissolved at $0.4 \mathrm{mM}$ in chloroform:methanol (2:1). The surface pressure-area ( $\pi-A)$ isotherms were recorded by means of an automated Langmuir trough (KSV Minitrough [width, $75 \mathrm{~mm}$; area, $24.225 \mathrm{~mm}^{2}$ ]; KSV Instruments) equipped with a platinum plate attached to a Wilhelmy-type balance. The GIPC sample was heated to $60^{\circ} \mathrm{C}$ for $15 \mathrm{~min}$ for a better solubilization. Pure solutions and lipid mixtures were spread (fixed volume of $30 \mu \mathrm{L}$ ) as tiny droplets to form a uniform monolayer on a Tris: $\mathrm{NaCl}$ 10:150 mM (Millipore) subphase adjusted to $\mathrm{pH} 7$ with $\mathrm{HCl}$. After evaporation of the solvent (15 min), monolayers were compressed at a rate of 5 $\mathrm{mm} / \mathrm{min}$ and at a temperature of $22^{\circ} \mathrm{C} \pm 1^{\circ} \mathrm{C}$. Before each experiment, the cleanliness of the system was confirmed by checking the surface pressure over the surface compression of the pure subphase. The reproducibility of the $\pi-A$ isotherms was checked by repeated recordings, and the relative SD in surface pressure and area was found to be $3 \%$ or less.

\section{Molecular modelling Approaches}

The Hypermatrix docking procedure was used to study the interaction of GIPC with the different sterols, as already described in (Cacas et al., 2016). Briefly, one GIPC molecule is positioned and fixed for the whole calculation at the centre of the system, oriented at the hydrophobic/hydrophilic interface. The interacting molecule is also oriented at the hydrophobic/hydrophilic interface, and by rotations and translations, more than 10 million positions of the interacting molecule around the central molecule are calculated. The lowest energy matching is considered as the most stable interaction. Refer to (Cacas et al., 2016) for more details.

\section{Liposomes preparation (Freeze and thaw method)}

The lipid solution of $1 \mathrm{mg} / \mathrm{ml}$ (GIPC/PLPC or POPC or DOPC/Stigmasterol or $\beta$ sitosterol) at different molar ratio, was dried and resuspended in water. Several cycles of freeze and thaw were done with freezing occurring in liquid nitrogen for 5 min and thawing at $50^{\circ} \mathrm{C}$ for $15 \mathrm{~min}$.

\section{LUV preparation for DLS and $\zeta-P o t e n t i a l$}

LUVs were prepared as described elsewhere,(Navon et al., 2017) with small modifications. Briefly, the lipid solution (GIPC/DOPC/Sterol) in $3 / 1 \mathrm{v} / \mathrm{v} \mathrm{THF} / \mathrm{H}_{2} \mathrm{O}$ 
methanol mixture was transferred into a round-bottom flask and the organic solvent was removed by evaporation under high vacuum pumping for $5 \mathrm{~h}$, until complete evaporation of the solvent. The lipid film was then hydrated in an appropriate amount of buffer solution and subjected to 3-5 freeze thaw cycles, yielding multilamellar vesicles. The resulting suspensions $\left(1 \mathrm{~g} \mathrm{~L}^{-1}\right)$ were then successively extruded 20 times through 200 and $100 \mathrm{~nm}$ polycarbonate membranes using a mini-extruder (Avanti Polar Lipids).

\section{DLS and $\zeta$-potential values}

Dynamic Light Scattering (DLS) and $\zeta$-Potential. DLS measurements were performed with a Malvern NanoZS instrument operating with a $2 \mathrm{~mW}$ HeNe laser at a wavelength of $632.8 \mathrm{~nm}$ and detection at an angle of $173^{\circ}$. All measurements were performed in a temperature-controlled chamber at $20^{\circ} \mathrm{C}\left( \pm 0.05^{\circ} \mathrm{C}\right)$. Three measurements of 15 runs each were usually averaged. The intensity size distribution was obtained from the analysis of the correlation function using the multiple narrow mode algorithm of the Malvern DTS software. The electrophoretic mobility of the vesicles and CNCs was measured by using the same Malvern NanoZS apparatus performing at $17^{\circ}$ from which the $\zeta$-potential values are determined by applying the Henry equation. The $\zeta$-potential values and the $\zeta$-deviation were averaged over at least three measurements with at least 30 runs per measurement. They were expressed as mean $\pm S D(n \geq 3)$.

\section{GUV preparation (Teflon method)}

GUV were prepared as previously described by Dimova (Kubsch et al., 2017). Briefly, $50 \mu \mathrm{L}$ of lipid mixture $\left(1 \mathrm{mg} \mathrm{mL}^{-1}\right)$ dissolved in organic solvent mixture were deposited on a pre-cleaned Teflon disk and the solvent was evaporated with vacuum for $2 \mathrm{hr}$. The disk was then placed in a $4 \mathrm{~mL}$ sealed glass vial with $200 \mathrm{mM}$ sucrose and $50 \mathrm{mM} \mathrm{NaCl}$ at $60^{\circ} \mathrm{C}$ for 12 hours, until a cloudy deposit was formed. For microscopy observation, one volume of the vesicle suspension was mixed with 4 volumes of iso-osmolar glucose/ $\mathrm{NaCl}$ solution for better contrast.

\section{Cryogenic Electronic Microscopy (Cryo-EM)}

Lacey carbon formvar 300mesh copper grids were used. They were first submitted to a standard glow discharged procedure (3mbar, 3mA for 40sec). Plunge freezing was realized using the EM-GP apparatus (Leica). Four microliters of the sample was deposited on the grid and immediately blotted for $2 \mathrm{sec}$ with a whatmann paper grade 5 before plunging into a liquid ethane bath cooled with liquid nitrogen $\left(-184^{\circ} \mathrm{C}\right)$. The settings of the chamber were fixed at $70 \%$ humidity and $20^{\circ} \mathrm{C}$. Total lipid concentration was $0.3 \mathrm{mg} / \mathrm{ml}$ Lipids molar ratio were as followed: POPC- ${ }^{2} \mathrm{H}_{31}$ / sterol (2:1), and GIPC/ POPC- ${ }^{2} \mathrm{H}_{31} /$ Sitosterol (1:1:1). Specimens were observed at $-170{ }^{\circ} \mathrm{C}$ using a cryo holder (626, Gatan, USA), with a ThermoFisher FEI Tecnai F20 electron microscope operating at $200 \mathrm{kV}$ under low-dose conditions. Images were acquired with an Eagle $4 \mathrm{k} \times 4 \mathrm{k}$ 
camera (ThermoFisher FEI) and processed in ImageJ. Deuterated POPC (POPC- $\left.{ }^{2} \mathrm{H}_{31}\right)$ were bought from Avanti and used as a marker for NMR measurements, GIPC were prepared from cauliflower. Sitosterol and stigmasterol were store bought from Avanti.

\section{Neutron Reflectivity}

Neutron reflectivity experiments were performed at the ILL, on the FIGARO reflectometer (Campbell et al., 2011), on SLBs formed through vesicle fusion on silicon crystals (Montis et al., 2016; Richter at al., 2006). The crystals (dimensions I $\times \mathrm{w} \times \mathrm{h}$ of $80 \times 50 \times 10 \mathrm{~mm}^{3}$ ) were polished through bath sonication in different solvents (5 min in chloroform; $5 \mathrm{~min}$ in acetone; $5 \mathrm{~min}$ in ethanol) followed by plasma cleaning. The substrates were then extensively rinsed with milliQ water and stored in milliQ water prior to use.

The specular reflectivity $(R)$ is defined as the ratio of reflected intensity over incident intensity of a neutron beam, when the angle of reflection is equal to the angle of incidence. It is measured from a flat surface using a highly collimated neutron beam as a function of momentum transfer $Q$, where $Q=4 \pi \sin \theta / \lambda$, with $\theta$ glancing angle and $\lambda$ wavelength. The measured reflectivity depends on the variation in the scattering length density profile, $\rho(z)$, perpendicular to the surface. The scattering length density profile over the z-axis was modeled as a sum of discrete contributions from separate layers, each characterized by a defined scattering length density, with a gaussian roughness contribution for each interface and a solvent penetration degree. The MOTOFIT software (Nelson, 2006) which runs in the IGOR Pro environment (http://www.wavemetrics.com), was used for the analysis of the NR curves.

A multilayer model was used to analyze the reflectivity profiles of the SLBs, with fixed scattering length density values calculated for each layer: (i) a first layer of a bulk subphase of $\mathrm{Si}\left(\rho=2.07 \times 10^{-6} \AA^{-2}\right)$ and a superficial layer of $\mathrm{SiO}_{2}\left(\rho=3.41 \times 10^{-6} \AA^{-2}\right)$, were introduced. Their thickness and interfacial roughness were characterized in control $\mathrm{NR}$ measurements in $\mathrm{D}_{2} \mathrm{O}$ and $\mathrm{H}_{2} \mathrm{O}$ before vesicle injection. (ii) The polar headgroups of the SLB of the inner and outer leaflet $\left(\rho=1.86 \times 10^{-6} \AA^{-2}\right)$ (iii) the bilayer lipid chains $(\rho$ $=-0.30 \times 10^{-6} \AA^{-2}$ ) (Wacklin, 2010) (iv) the sugar heads of the GIPC were represented as additional layer to the phosphate polar head group in the inner and outer leaflets $(\rho=$ $1.9 \times 10^{-6} \AA^{-2}$ ). (v) finally, a bulk super phase of solvent was introduced to the model.

All measurements were performed in four contrast solvents, namely $\mathrm{H}_{2} \mathrm{O}(\rho=-0.56 \times$ $\left.10^{-6} \AA^{-2}\right), D_{2} \mathrm{O}\left(\rho=6.34 \times 10^{-6} \AA^{-2}\right), 4 \mathrm{MW}\left(34 \% \mathrm{H}_{2} \mathrm{O}\right.$ and $\left.66 \% \mathrm{D}_{2} \mathrm{O}, \rho=4.0 \times 10^{-6} \AA^{-2}\right)$, or SMW $\left(62 \% \mathrm{H}_{2} \mathrm{O}\right.$ and $\left.38 \% \mathrm{D}_{2} \mathrm{O}, \rho=2.07 \times 10^{-6} \AA^{-2}\right)$.

\section{Solid State ${ }^{2} \mathrm{H}-\mathrm{NMR}$}


Samples were prepared by co-solubilizing the appropriate amount of Bo-GIPC, $\mathrm{POPC}^{2} \mathrm{H}_{31}$, sitosterol and stigmasterol in chloroform. Solvent was evaporated under a flow of nitrogen to obtain a thin lipid film, rehydrated with ultra-pure water before onenight lyophilization. The lipid powder was finally hydrated with $100 \mu \mathrm{l}$ of deuteriumdepleted water (hydration of 97\%). Samples were transferred into 100 $\mu$ l 4-mm zirconia rotors for NMR analyses. ${ }^{2} \mathrm{H}$-ssNMR experiments were performed at $76.77 \mathrm{MHz}$ with a

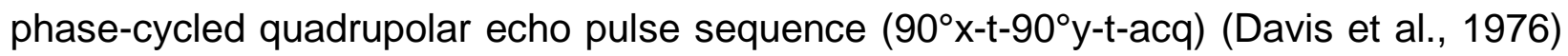
and using a Bruker Avance III $500 \mathrm{MHz}$ WB (11.75 T) spectrometer equipped with a solid state CPMAS 4mm H/F/X probe (IECB structural biophysics platform, Bordeaux, France). Acquisition parameters were as follows: spectral window of $500 \mathrm{kHz}, \pi / 2$ pulse width of $3.5 \mu \mathrm{s}$, interpulse delays of $40 \mu \mathrm{s}$, recycling delay of $2 \mathrm{~s}$; number of scans from $1 \mathrm{~K}$ to $6 \mathrm{~K}$. Spectra were processed using a Lorentzian line broadening of $300 \mathrm{~Hz}$ before Fourier transformation from the top of the echo. Samples were equilibrated for $20 \mathrm{~min}$ at a given temperature before data acquisition. All spectra were processed and analyzed using Bruker Topspin 4.0.6 software. First moments were calculated using a $\mathrm{C}^{2+}$ homemade routine (Buchoux S., unpublished).

\section{LC-MS analysis}

For the analysis of sphingolipids by LC-MS/MS, lipids extracts were then incubated $1 \mathrm{~h}$ at $50^{\circ} \mathrm{C}$ in $2 \mathrm{~mL}$ of methylamine solution $(7 \mathrm{ml}$ methylamine $33 \%(\mathrm{w} / \mathrm{v})$ in $\mathrm{EtOH}$ combined with $3 \mathrm{~mL}$ of methylamine $40 \%(\mathrm{w} / \mathrm{v})$ in water (Sigma Aldrich) in order to remove phospholipids. After incubation, methylamine solutions dried at $40^{\circ} \mathrm{C}$ under a stream of air (Markham \& Jaworski, 2007). Finally, were resuspended into $100 \mu \mathrm{L}$ of $\mathrm{THF} / \mathrm{MeOH} / \mathrm{H} 2 \mathrm{O}(40: 20: 40$, v/v) with $0.1 \%$ formic acid containing synthetic internal lipid standards (Cer d18:1/C17:0, GluCer d18:1/C12:0 and GM1) was added, thoroughly vortexed, incubated at $60^{\circ} \mathrm{C}$ for $20 \mathrm{~min}$, sonicated $2 \mathrm{~min}$ and transferred into LC vials. LC-MS/MS (multiple reaction monitoring mode) analyses were performed with a model QTRAP 6500 (ABSciex) mass spectrometer coupled to a liquid chromatography system (1290 Infinity II, Agilent). Analyses were performed in the positive mode. Nitrogen was used for the curtain gas (set to 30 ), gas 1 (set to 30), and gas 2 (set to 10). Needle voltage was at $+5500 \mathrm{~V}$ with needle heating at $400^{\circ} \mathrm{C}$; the declustering potential was adjusted between +10 and $+40 \mathrm{~V}$. The collision gas was also nitrogen; collision energy varied from +15 to $+60 \mathrm{eV}$ on a compound-dependent basis. Reverse-phase separations were performed at $40^{\circ} \mathrm{C}$ on a Supercolsil $\mathrm{ABZ}+, 100 \times 2.1 \mathrm{~mm}$ column and $5 \mu \mathrm{m}$ particles (Supelco). The Eluent $A$ was THF/ACN/5 mM Ammonium formate (3/2/5 $\mathrm{v} / \mathrm{v} / \mathrm{v}$ ) with $0.1 \%$ formic acid and eluent B was THF/ACN/5 mM Ammonium formate $(7 / 2 / 1 \mathrm{v} / \mathrm{v} / \mathrm{v})$ with $0.1 \%$ formic acid. The gradient elution program for Cer and GluCer quantification was as follows: 0 to $1 \mathrm{~min}, 1 \%$ eluent B; $40 \mathrm{~min}, 80 \%$ eluent $\mathrm{B}$; and 40 to $42,80 \%$ eluent $\mathrm{B}$. The gradient elution program for GIPC quantification was as follows: 0 to $1 \mathrm{~min}, 15 \%$ eluent B;31 $\mathrm{min}, 45 \%$ eluent B; $47.5 \mathrm{~min}, 70 \%$ eluent B; and 47.5 to 49 , 
$70 \%$ eluent $\mathrm{B}$. The flow rate was set at $0.2 \mathrm{~mL} / \mathrm{min}$, and $5 \mathrm{~mL}$ sample volumes were injected. The areas of LC peaks were determined using MultiQuant software (version 3.0; ABSciex) for sphingolipids quantification, see supplemental table 1 the list of molecules $\mathrm{Q} 1$ ions and $\mathrm{Q} 3$ ions. 


\section{Acknowledgements}

We thank Jean-Paul Douliez, Catherine Sarazin and Sébastien Buchoux for critical reading and advice and Laure Beven for bright field microscopy. We thank Claire Bréhélin for the help in electronic microscopy observations. M.D. and L.L. thank the FRS-FNRS for their position as Senior Research Associates. We benefited from the facilities and expertises of the Biophysical and Structural Chemistry platform (BPCS) at IECB, CNRS UMS3033, Inserm US001, Bordeaux University http://www.iecb.ubordeaux.fr/index.php/fr/plateformestechnologiques. FS, SM, LL, LF DB are funded by the ANR PlayMobil (ANR-19-CE20-0016-02). YG, JCM were funded as was part of the DOE Joint BioEnergy Institute (http://www.jbei.org) supported by the U. S. Department of Energy, Office of Science, Office of Biological and Environmental Research, through contract DE-AC02-05CH11231 between Lawrence Berkeley National Laboratory and the U. S. Department of Energy. We thank BordeauxMetabolome platform for lipid analysis supported by ANR PlayMobil (grant no. ANR-19CE20-0016-02 to S.M., L.F., F.S.-P. and Bordeaux Metabolome Facility-MetaboHUB (grant no. ANR-11-INBS-0010 to S.M. and L.F.). 


\section{Figure legends}

\section{Figure 1}

A, Structure of GIPC series A (2 sugars after the inositol group). B, GIPC content of different plant species: Brassica oleracea (cauliflower), Nicotiana tabacum (BY-2 cell culture), Allium porrum (leek) and Oryza sativa (rice cell culture). The GIPC content in $\mathrm{mg}$ per $\mathrm{g}$ of fresh weight was estimated by calculating the proportion of (h)VLCFA (hydroxylated Very Long Chain Fatty Acid) as determined by fatty acid methyl ester (GC-MS). The type of GIPC was defined by HPTLC analysis based on Cacas et al., 2016. Three to five independent samples were processed.

\section{Figure 2}

Extraction and purification protocol of GIPCs. GIPC purification scheme, adapted from (Carter \& Koob, 1969; Kaul \& Lester, 1975; Markham \& Jaworski, 2007). The three steps labelled 1, 2 and 3, respectively are important milestones in the GIPC isolation steps.

\section{Figure 3}

Gas Chromatography- Mass Spectrometry (GC-MS) analysis of fatty acid content after steps $\alpha, \beta$ and $y$ of the extraction and purification process (see Figure 2) (A) and HighPerformance Thin Layer Chromatography (HPTLC) assay of lipid content after step 3 (B). A, Aliquots of (I) Bo-cauliflower, (II) Nt-BY-2, (III) Ap-leek and (IV) Os-rice samples at step 1,2 and 3 underwent trans-methylation to release fatty acid before derivatization by BSTFA, the resulting FAMES were analysed by GC-MS and the fatty acid content calculated $(n=3)$. FA refer to fatty acid of 16 to 18 carbon atoms fatty acids and (h)VLCFA refer to hydroxylated or non-hydroxylated very long chain fatty acid of 20 to 28 carbon atoms. The amount of GIPC in each sample were extrapolated from the (h)VLCFA content. Data shown for 3 independent replicas. Error bars are SD. B, HPTLC assay shows the lipid content of GIPC-enriched samples after step 3. Cauliflower (I) contains mainly GIPC series A, BY-2 (Nt-GIPC) sample (II) were further separated by column chromatography to isolate the different GIPC series. Nt $(\alpha)$ GIPCenriched sample contains mainly series $\mathrm{A}, \mathrm{B}$ and $\mathrm{C}$ while $\mathrm{Nt}(\beta)$ and $\mathrm{Nt}(\gamma)$ show presence of polyglycosylated GIPCs (series D, E ,F, etc). Leek (III) and rice (IV) samples contain mainly GIPC series B.

\section{Figure 4}

A, Very long-chain fatty acid (VLCFA) and hydroxylated VLCFA (hVLCFA) content of GIPC enriched samples from cauliflower, BY-2 cell culture, leek and rice cell culture. The fatty acids were released from the GIPC enriched samples by transmethylation followed by derivatization using BSTFA, before GC-MS analysis. B, HPAEC analysis of GIPC-enriched samples shows the monosaccharide content after TFA hydrolysis. Abbreviations are as follows: GlcA: glucuronic acid; Glc: glucose; GlcN: glucosamine; Man: mannose; Gal: galactose; Ara: arabinose; Xyl: xylose; Fuc: fucose; Rha: rhamnose; GalA: galacturonic acid. C, Yariv reactivity test of GIPC-enriched samples to detect arabino-galactan content. No arabino-galactan were detected. 50ug of each 
sample $(1 \mathrm{mg} / \mathrm{ml})$ was deposited in each well, the picture was taken $48 \mathrm{~h}$ after initiating the reaction.

\section{Figure 5}

Surface pressure-area ( $\pi-A)$ isotherms, at the air-aqueous phase interface, of pure GIPC and sterol monolayers and of mixed GIPC/sterol monolayer prepared at a molar ratio of 0.80 . A. The isotherms were recorded at $25^{\circ} \mathrm{C}$ on an aqueous subphase composed by $10 \mathrm{mM}$ Tris buffer at $\mathrm{pH}$ 7. Duplicate experiments using independent preparations yielded similar results. B, Comparison of the experimental (blue bars) and theoretical (red bars) mean molecular areas at a surface pressure of $30 \mathrm{mN} / \mathrm{m}$ for a GIPC/sterol molar ratio of 0.80 . The theoretical value is obtained according to the additivity rule: $A_{12}=A_{1} X_{1}+A_{2} X_{2}$, where $A_{12}$ is the mean molecular area for ideal mixing of the two components at a given $\pi, A_{1}$ and $A_{2}$ are the molecular areas of the respective components in their pure monolayers at the same $\pi$, and $X_{1}$ and $X_{2}$ are the molar ratios of components 1 and 2 in the mixed monolayers. C, Excess free energy of mixing ( $\Delta \mathrm{Gex}$; blue bars) and free energy of mixing ( $\Delta \mathrm{GM}$; red bars) of the mixed monolayer GIPC/sterol at a molar ratio of 0.80 at the surface pressure of $30 \mathrm{mN} / \mathrm{m}$. $\Delta$ Gex and $\Delta \mathrm{GM}$ were calculated according to the equations as shown in (Eeman et al., 2005; MagetDana, 1999). Abbreviations are as follows: SG, steryl glucoside (sitosterol, glucose head group); ASG, acyl steryl glucoside (sitosterol, glucose head group and C18:2 acyl chain). Error bars are SD.

\section{Figure 6}

Modelling of the interaction of GIPC and sterols. Theoretical interactions calculated by HyperMatrix docking method with one molecule of GIPC serie A t18:0/h24:0 and one molecule of either A, $\beta$-sitosterol, or B, Stigmasterol or C, Steryl Glucoside, SG ( $\beta$ sitosterol, with glucose head group), or D Acyl Steryl Glucoside, ASG ( $\beta$-sitosterol, with glucose head group/18:2 acyl chain).

\section{Figure 7}

A, Bo-GIPC containing-liposomes in buffer solution after 3 cycles of freeze and thaw. Enriched Bo-GIPC (cauliflower), underwent freeze $\left(-20^{\circ} \mathrm{C}, 20 \mathrm{~min}\right)$ and thaw $\left(60^{\circ} \mathrm{C}, 20\right.$ min) cycles three times GIPC in TBS buffer $\mathrm{pH} 5,8$ with or without phospholipid and $\beta$ sitosterol at a concentration of $1 \mathrm{mg} / \mathrm{ml}$. (I) GIPCs alone form crystals in a saline buffer solution. A lipid mix, at a concentration of $1 \mathrm{mg} / \mathrm{ml}$, of GIPC/PLPC/ $\beta$-sitosterol or GIPC/POPC/ $\beta$-sitosterol $(1: 1: 1, \mathrm{~mol} / \mathrm{mol})$, shown in (II) and (III) respectively, forms vesicles of approx. $2 \mu \mathrm{m}$. B, Fluorescence and phase contrast microscopy images of Giant unilamellar vesicles (GUVs) of GIPC/DOPC/ $\beta$-sitosterol $(1: 1: 1, \mathrm{~mol} / \mathrm{mol})$. The lipid mix was labled by NBD-PC at $0.1 \%$. mol. C, Dynamic light scattering (DLS) and $\zeta$ potential of liposomes containing DOPC/ $\beta$-sitosterol (7:3, mol ratio) (yellow) and GIPC/DOPC/ $\beta$-sitosterol (1:1:1, mol ratio) (green), respectively provide the size which is around $100 \mathrm{~nm}$ and $\zeta$-potential values of $-28 \mathrm{mV}$ in the presence of GIPC. Error bars are SD.

\section{Figure 8}


Liposomes shapes varies with lipid composition. A, Cryo-EM images of liposomes. POPC-d31 that is a deuterated POPC on the carbon of the palmitoyl chain: 16:0-d3118:1 PC, in the presence of sterols (ii sitosterol and iv: stigmasterol) are mainly present as vesicles, showing one to few bilayers. In the presence of GIPC, these liposomes are still observed but at the same time, rigid bilayers structures appearing as flat entities are also observed (white arrows in I and iii). Scale bar, $100 \mathrm{~nm}$. B. membrane thickness measurements. Measurements were made using ImageJ software to compare the membrane thickness with or without GIPC. Error bars are SD. Significance was determined by Student's $t$-test. ${ }^{* * *} \mathrm{P}<0.0001$

\section{Figure 9}

Reflectivity profiles and calculated Scattering length density (SLD) following lipid bilayer deposition of (i) POPC/ $\beta$-sitosterol $(70: 30, \mathrm{~mol} / \mathrm{mol}$ ) and (ii) GIPC/POPC/ $\beta$-sitosterol $(55: 15: 30, \mathrm{~mol} / \mathrm{mol})$. A, The multilayer model was composed from the silicon substrate $\left(S L D=2.0710^{-6} \AA^{-2}\right)$ covered with a layer of silicon oxide $\left(S L D=3.4710^{-6} \AA^{-2}\right) . B$, Structural parameters after multilayer model fitting of reflectivity profiles of lipid bilayer. $\mathrm{C}$, Scheme showing the SLD profile overlaid on the multilayer model as obtained for POPC membrane.

\section{Figure 10}

A, ${ }^{2} \mathrm{H}$-NMR powder spectra of lipid mix and B, First spectral moment of ${ }^{2} \mathrm{H}-\mathrm{NMR}$ spectra showing membrane ordering vs. temperature $\mathrm{POPC}-{ }^{2} \mathrm{H}_{31}$ system alone, in binary systems of POPC- ${ }^{2} \mathrm{H}_{31} / \beta$-sitosterol $(1: 1 \mathrm{~mol} / \mathrm{mol})$ and $\mathrm{POPC}-{ }^{2} \mathrm{H}_{31} /$ Stigmasterol $(1: 1$ $\mathrm{mol} / \mathrm{mol}$ ) and ternary systems of GIPC/ POPC- ${ }^{2} \mathrm{H}_{31} / \beta$-sitosterol $(1: 1: 1 \mathrm{~mol}$ ratio) and GIPC/ POPC- ${ }^{2} \mathrm{H}_{31} /$ Stigmasterol (1:1:1 mol ratio). 


\section{Supplementary data Figure legends}

\section{Supplementary data 1}

High performance thin layer chromatography (HPTLC) assay of eluted fractions collected during the GIPC purification process (described in Figure 2). \#2 refers to the crude extract deposited on the silica column. Fractions containing GIPC without visible contamination of sterols and phospholipids were selected and pooled to make up fraction \#3.

\section{Supplementary data 2}

Fatty acid contents of GIPC-enriched fractions purified from cauliflower, BY-2 cell culture, leek and rice cell culture. The fatty acid content was quantified after releasing fatty acid by transmethylation of GIPC-enriched samples, followed by derivatization by BSTFA before GC-MS analysis. The most abundant pool of fatty acid are hydroxylated fatty acid $>20$ carbons (hVLCFA) for Bo-GIPC and Nt-GIPC enriched-samples and nonhydroxylated fatty acid $>20$ carbons (VLCFA) for Ap-GIPC and Os-GIPC enrichedsample. The data are means of three independent experiments. Error bars are SD.

\section{Supplementary data 3}

Determining glycan content by HPAE analysis of GIPC-enriched samples. A slight change in sugar amount was seen after $1 \mathrm{~h}, 3 \mathrm{~h}$ and $4 \mathrm{~h}$ of TFA hydrolysis. (GlcA: glucuronic acid; Glc: glucose; GlcN: glucosamine; Man: mannose; Gal: galactose; Ara: arabinose; Xyl: xylose; Fuc: fucose; Rha: rhamnose; GalA: galacturonic acid).

\section{Supplementary data 4}

LC-MS-based sphingolidomic showing the LCB, gluCER, and GIPC content found in crude extract of cauliflower and leek, and purified Bo-GIPC and Ap-GIPC. Analysis were performed on three independent purifications and expressed as the mean relative amount in percentage of the three analysis.

\section{Supplementary data 5}

LC-MS-based sphingolidomic showing the different molecular species found in crude extract of cauliflower and leek, and purified Bo-GIPC and Ap-GIPC according to the LCB or (very long chain) fatty acid (VLC)FA content. Analysis were performed on triplicate of three independent purifications. The mean relative amount of each species were calculated and expressed in percentage..

\section{Supplementary data 6}

Fluorescent microscopy observations of Nt-GIPC containing liposomes in water at RT, $\mathrm{pH}$ 7. A, Liposomes obtained after 3 cycles of freeze in liquid nitrogen and thaw (water bath at $60^{\circ} \mathrm{C}$ ) containing (I) Nt-GIPCs $2 \mathrm{mg} / \mathrm{ml}$; (II) Nt-GIPC/ 1,2-dimyristoyl-sn-glycero3-phosphocholine (DMPC) $(4: 1 \mathrm{~mol} / \mathrm{mol})$ at $2 \mathrm{mg} / \mathrm{ml}$; (III) Nt-GIPC/ DMPC $(1: 1 \mathrm{~mol} / \mathrm{mol})$ at $2 \mathrm{mg} / \mathrm{ml}$ shows crystals; (IV) Nt-GIPC/DMPC molar ratio $(1: 4 \mathrm{~mol} / \mathrm{mol})$ at $2 \mathrm{mg} / \mathrm{ml}$ forms liposomes of $10 \mu \mathrm{m}$. (V) HPTLC analysis of lipid mixture confirms the presence of 
GIPC and DMPC in the liposome mix observed. The higher the GIPC content, the higher the occurence of crystal formation. GIPCs can form liposomes with phospholipids of short acyl chains when the latter is four-folds more abundant in the mix. Scale bar, $5 \mu \mathrm{m}$. B, Liposomes of Bo-cauliflower GIPC/POPC/ $\beta$-sitosterol $(1: 1: 1 \mathrm{~mol} / \mathrm{mol})$ at $1 \mathrm{mg} / \mathrm{ml}$ in TBS $1 X$. Clusters as shown in (I) were obtained after three cycles of $20 \mathrm{~min}$ freezing at $-20^{\circ} \mathrm{C}$ and thawing in a water bath at $60^{\circ} \mathrm{C}$. Liposomes in (II) were formed after three cycles of freezing in liquid $\mathrm{N}_{2}$ and 20 min heating in a water bath at $60^{\circ} \mathrm{C}$; no cluster was observed. The type of freeze/thaw determines the size and shape of liposomes formed. Scale bar, $5 \mu \mathrm{m}$. 


\section{References}

Baumgart, T., Hunt, G., Farkas, E. R., Webb, W. W., \& Feigenson, G. W. (2007). Fluorescence probe partitioning between Lo/Ld phases in lipid membranes. Biochimica et Biophysica Acta - Biomembranes, 1768(9), 2182-2194. https://doi.org/10.1016/j.bbamem.2007.05.012

Beck, J. G., Mathieu, D., Loudet, C., Buchoux, S., \& Dufourc, E. J. (2007). Plant sterols in "rafts": A better way to regulate membrane thermal shocks. FASEB Journal, 21(8), 1714-1723. https://doi.org/10.1096/fj.06-7809com

Buré, C., Cacas, J. L., Badoc, A., Mongrand, S., \& Schmitter, J. M. (2016). Branched glycosylated inositolphosphosphingolipid structures in plants revealed by MS3analysis. Journal of Mass Spectrometry. https://doi.org/10.1002/jms.3758

Buré, C., Cacas, J. L., Mongrand, S., \& Schmitter, J. M. (2014). Characterization of glycosyl inositol phosphoryl ceramides from plants and fungi by mass spectrometry. Analytical and Bioanalytical Chemistry, Vol. 406, pp. 995-1010. https://doi.org/10.1007/s00216-013-7130-8

Buré, C., Cacas, J. L., Wang, F., Gaudin, K., Domergue, F., Mongrand, S., \& Schmitter, J. M. (2011). Fast screening of highly glycosylated plant sphingolipids by tandem mass spectrometry. Rapid Communications in Mass Spectrometry. https://doi.org/10.1002/rcm.5206

Cacas, J. L., Buré, C., Furt, F., Maalouf, J. P., Badoc, A., Cluzet, S., ... Mongrand, S. (2013). Biochemical survey of the polar head of plant glycosylinositolphosphoceramides unravels broad diversity. Phytochemistry. https://doi.org/10.1016/j.phytochem.2013.08.002

Cacas, J. L., Buré, C., Grosjean, K., Gerbeau-Pissot, P., Lherminier, J., Rombouts, Y., ... Mongrand, S. (2016). Revisiting plant plasma membrane lipids in tobacco: A focus on sphingolipids. Plant Physiology, 170(1), 367-384. https://doi.org/10.1104/pp.15.00564

Campbell, R. A., Wacklin, H. P., Sutton, I., Cubbit, R., \& Fragneto, G. (2011). FIGARO $\square$ : The new horizontal neutron reflectometer at the ILL. European Physical Journal Plus, (November). https://doi.org/10.1140/epjp/i2011-11107-8

Carter, H. E., Gigg, R. H., Law, J. H., Nakayama, T., \& Weber, E. (1958). Biochemistry of the sphingolipides. XI. Structure of phytoglycolipide. The Journal of Biological Chemistry, 233(6), 1309-1314. Retrieved from http://www.jbc.org/

Carter, H. E., \& Kisic, A. (1969). Countercurrent distribution of inositol lipids of plant seeds. J. Lipid Res, 10(4)(Jul), 363-369.

Carter, H. E., \& Koob, J. L. (1969). Sphingolipids in bean leaves (Phaseolus vulgaris). Journal of Lipid Research, 10(4), 363-369.

Courtney, K. C., Fung, K. Y. Y., Maxfield, F. R., \& Fairn, G. D. (2018). Comment on ‘ 
Orthogonal lipid sensors identify transbilayer asymmetry of plasma membrane cholesterol .' ELife, 1-8.

Davis, J. H. (1983). The description of membrane lipid conformation, order and dynamics byn 2H-NMR. Biochimica et Biophysica Acta (BBA), 737, 117-171.

Davis, J. H., Jeffrey, K. R., Bloom, M., \& Valic, M. . (1976). Quadrupolar Echo deuteron magnetic resonance spectroscopy in ordered hydrocarbon chains. Chemical Physics Letters, 42(2), 390-394.

Deleu, M., Crowet, J. M., Nasir, M. N., \& Lins, L. (2014). Complementary biophysical tools to investigate lipid specificity in the interaction between bioactive molecules and the plasma membrane: A review. Biochimica et Biophysica Acta Biomembranes, 1838(12), 3171-3190. https://doi.org/10.1016/j.bbamem.2014.08.023

Eeman, M., Deleu, M., Paquot, M., Thonart, P., \& Dufre, Y. F. (2005). Nanoscale Properties of Mixed Fengycin / Ceramide Monolayers Explored Using Atomic Force Microscopy. Langmuir, (16), 2505-2511. https://doi.org/10.1021/la0475775

Fang, L., Ishikawa, T., Rennie, E. A., Murawska, G. M., Lao, J., Yan, J., ... Mortimer, J. C. (2016). Loss of Inositol Phosphorylceramide Sphingolipid Mannosylation Induces Plant Immune Responses and Reduces Cellulose Content in Arabidopsis. The Plant Cell. https://doi.org/10.1105/tpc.16.00186

Furt, F., Lefebvre, B., Cullimore, J., Bessoule, J. J., \& Mongrand, S. (2007). Plant lipid rafts: Fluctuat nec mergitur. Plant Signaling and Behavior, 2(6), 508-511. https://doi.org/10.4161/psb.2.6.4636

Gerbeau-Pissot, P., Der, C., Thomas, D., Anca, I. A., Grosjean, K., Roche, Y., ... Simon-Plas, F. (2014). Modification of plasma membrane organization in tobacco cells elicited by cryptogein. Plant Physiology, 164(1), 273-286. https://doi.org/10.1104/pp.113.225755

Goldenberg, N. M., \& Steinberg, B. E. (2010, February 15). Surface charge: A key determinant of protein localization and function. Cancer Research, Vol. 70, pp. 1277-1280. https://doi.org/10.1158/0008-5472.CAN-09-2905

Gronnier, J., Gerbeau-Pissot, P., Germain, V., Mongrand, S., \& Simon-Plas, F. (2018, October 1). Divide and Rule: Plant Plasma Membrane Organization. Trends in Plant Science, Vol. 23, pp. 899-917. https://doi.org/10.1016/j.tplants.2018.07.007

Grosjean, K., Mongrand, S., Beney, L., Simon-Plas, F., \& Gerbeau-Pissot, P. (2015). Differential effect of plant lipids on membrane organization specificities of phytosphingolipids and phytosterols. Journal of Biological Chemistry, 290(9), 58105825. https://doi.org/10.1074/jbc.M114.598805

Gutierrez, A. L. S., Farage, L., Melo, M. N., Mohana-Borges, R. S., Guerardel, Y., Coddeville, B., ... Guerardel, Y. (2007). Characterization of glycoinositolphosphoryl 
ceramide structure mutant strains of Cryptococcus neoformans. Glycobiology. https://doi.org/10.1093/glycob/cwm030

Hsieh, T. C., Lester, R. L., \& Laine, R. A. (1981). Glycophosphoceramides from plants. Purification and characterization of a novel tetrasaccharide derived from tobacco leaf glycolipids. Journal of Biological Chemistry.

Ishikawa, T., Fang, L., Rennie, E. A., Sechet, J., Yan, J., Jing, B., ... Mortimer, J. C. (2018). GLUCOSAMINE INOSITOLPHOSPHORYLCERAMIDTRANSFERASE1 (GINT1) is a GlcNAc-containing glycosylinositol phosphorylceramide glycosyltransferase. Plant Physiology, 177(3), 938-952. https://doi.org/10.1104/pp.18.00396

Jiang, Z., Zhou, X., Tao, M., Yuan, F., Liu, L., Wu, F., ... Pei, Z.-M. (2019). Plant cellsurface GIPC sphingolipids sense salt to trigger $\mathrm{Ca} 2+$ influx. Nature. https://doi.org/10.1038/s41586-019-1449-z

Kaul, K., \& Lester, R. L. (1975). Characterization of Inositol-containing Phosphosphingolipids from Tobacco Leaves. Plant Physiology, 55(1), 120-129. https://doi.org/10.1104/pp.55.1.120

Kaul, K., \& Lester, R. L. (1978). Isolation of Six Novel Phosphoinositol-Containing Sphingolipids from Tobacco Leaves. Biochemistry, 17(17), 3569-3575. https://doi.org/10.1021/bi00610a023

Kitazawa, K., Tryfona, T., Yoshimi, Y., Hayashi, Y., Kawauchi, S., Antonov, L., ... Kotake, T. (2013). $\beta$-Galactosyl Yariv Reagent Binds to the $\beta-1,3-$ Galactan of Arabinogalactan Proteins. Plant Physiology, 161(3), 1117-1126. https://doi.org/10.1104/pp.112.211722

Koynova, R., \& Caffrey, M. (1998). Phases and phase transitions of the phosphatidylcholines. Biochimica et Biophysica Acta, 1378.

Kubsch, B., Robinson, T., Steinkühler, J., \& Dimova, R. (2017). Phase behavior of charged vesicles under symmetric and asymmetric solution conditions monitored with fluorescence microscopy. Journal of Visualized Experiments, 2017(128). https://doi.org/10.3791/56034

Lefebvre, B., Furt, F., Hartmann, M.-A., Michaelson, L. V., Carde, J.-P., Sargueil-Boiron, F., ... Mongrand, S. (2007). Characterization of Lipid Rafts from Medicago truncatula Root Plasma Membranes: A Proteomic Study Reveals the Presence of a Raft-Associated Redox System. PLANT PHYSIOLOGY, 144(1), 402-418. https://doi.org/10.1104/pp.106.094102

Lenarčič, T., Albert, I., Böhm, H., Hodnik, V., Pirc, K., Zavec, A. B., ... Nürnberger, T. (2017). Eudicot plant-specific sphingolipids determine host selectivity of microbial NLP cytolysins. Science, 358(6369), 1431-1434. https://doi.org/10.1126/science.aan6874 
Levental, I., \& Veatch, S. L. (2016, December 4). The Continuing Mystery of Lipid Rafts. Journal of Molecular Biology, Vol. 428, pp. 4749-4764. https://doi.org/10.1016/j.jmb.2016.08.022

Lingwood, D., \& Simons, K. (2010, January 1). Lipid rafts as a membrane-organizing principle. Science, Vol. 327, pp. 46-50. https://doi.org/10.1126/science.1174621

London, E., \& Brown, D. A. (2000). Insolubility of lipids in Triton X-100: physical origin and relationship to sphingolipid/cholesterol membrane domains (rafts). Biochimica et Biophysica Acta (BBA) - Biomembranes, 1508(1-2), 182-195. https://doi.org/10.1016/S0304-4157(00)00007-1

Luttgeharm, K. D., Kimberlin, A. N., Cahoon, R. E., Cerny, R. L., Napier, J. A., Markham, J. E., \& Cahoon, E. B. (2015). Sphingolipid metabolism is strikingly different between pollen and leaf in Arabidopsis as revealed by compositional and gene expression profiling. Phytochemistry, 115(1), 121-129.

https://doi.org/10.1016/j.phytochem.2015.02.019

Maget-Dana, R. (1999). The monolayer technique: A potent tool for studying the interfacial properties of antimicrobial and membrane-lytic peptides and their interactions with lipid membranes. In Biochimica et Biophysica Acta Biomembranes (Vol. 1462). https://doi.org/10.1016/S0005-2736(99)00203-5

Mamode Cassim, A., Gouguet, P., Gronnier, J., Laurent, N., Germain, V., Grison, M., ... Mongrand, S. (2019). Plant lipids: Key players of plasma membrane organization and function. Progress in Lipid Research, 73(November 2018), 1-27. https://doi.org/10.1016/j.plipres.2018.11.002

Mannock, D. A., Lewis, R. N. A. H., McMullen, T. P. W., \& McElhaney, R. N. (2010, June). The effect of variations in phospholipid and sterol structure on the nature of lipid-sterol interactions in lipid bilayer model membranes. Chemistry and Physics of Lipids, Vol. 163, pp. 403-448. https://doi.org/10.1016/j.chemphyslip.2010.03.011

Markham, J. E., \& Jaworski, J. G. (2007). Rapid measurement of sphingolipids from Arabidopsis thaliana by reversed-phase high-performance liquid chromatography coupled to electrospray ionization tandem mass spectrometry. Rapid Communications in Mass Spectrometry. https://doi.org/10.1002/rcm.2962

Marsh, D. (1996). Lateral pressure in membranes. 1286.

Mongrand, S., Morel, J., Laroche, J., Claverol, S., Carde, J. P., Hartmann, M. A., ... Bessoule, J. J. (2004). Lipid rafts in higher plant cells: Purification and characterization of triton X-100-insoluble microdomains from tobacco plasma membrane. Journal of Biological Chemistry, 279(35), 36277-36286. https://doi.org/10.1074/jbc.M403440200

Montis, C., Gerelli, Y., Fragneto, G., Nylander, T., Baglioni, P., \& Berti, D. (2016). Nucleolipid bilayers $\square$ : A quartz crystal microbalance and neutron reflectometry study. Colloids and Surfaces B $\square$ : Biointerfac, 137, 203-213. 
Moreau, R. A., Nyström, L., Whitaker, B. D., Winkler-Moser, J. K., Baer, D. J., Gebauer, S. K., \& Hicks, K. B. (2018, April 1). Phytosterols and their derivatives: Structural diversity, distribution, metabolism, analysis, and health-promoting uses. Progress in Lipid Research, Vol. 70, pp. 35-61. https://doi.org/10.1016/j.plipres.2018.04.001

Morikawa, T., Mizutani, M., Aoki, N., Watanabe, B., Saga, H., Saito, S., ... Ohta, D. (2006). Cytochrome P450 CYP710A encodes the sterol C-22 desaturase in Arabidopsis and tomato. Plant Cell, 18(4), 1008-1022. https://doi.org/10.1105/tpc.105.036012

Morré, D. M., \& Morre, D. J. (2000). Aqueous two-phase partition applied to the isolation of plasma membranes and Golgi apparatus from cultured mammalian cells. Journal of Chromatography B: Biomedical Sciences and Applications, 743(1-2), 377-387. https://doi.org/10.1016/S0378-4347(00)00058-X

Mortimer, J. C., \& Scheller, H. V. (2020). Trends in Plant Science Synthesis and Function of Complex Sphingolipid Glycosylation Trends in Plant Science. Trends in Plant Science, 25(6), 522-524. https://doi.org/10.1016/j.tplants.2020.03.007

Mortimer, J. C., Yu, X., Albrecht, S., Sicilia, F., Huichalaf, M., Ampuero, D., ... Dupree, P. (2013). Abnormal glycosphingolipid mannosylation triggers salicylic acidmediated responses in Arabidopsis. Plant Cell, 25(5), 1881-1894. https://doi.org/10.1105/tpc.113.111500

Nagano, M., Ishikawa, T., Fujiwara, M., Fukao, Y., \& Kawano, Y. (2016). Plasma Membrane Microdomains Are Essential for Rac1-RbohB / H-Mediated Immunity in Rice. The Plant Cell, 28(August), 1966-1983. https://doi.org/10.1105/tpc.16.00201

Navon, Y., Radavidson, H., Putaux, J., Jean, B., \& Heux, L. (2017). pH-Sensitive Interactions between Cellulose Nanocrystals and DOPC Liposomes. Biomacromolecules, 18, 2918-2927. https://doi.org/10.1021/acs.biomac.7b00872

Nelson, A. (2006). Co-refinement of multiple-contrast neutron / X-ray reflectivity data using MOTOFIT. Journal of Applied Crystallography, 39, 273-276. https://doi.org/10.1107/S0021889806005073

Oldfield, E., Meadows, M., Rice, D., \& Jacobs, R. (1978). Spectroscopic Studies of Specifically Deuterium Labeled Membrane Systems . Nuclear Magnetic Resonance Investigation of the Effects of Cholesterol in Model Systems '. American Chemical Society, 17(14).

Pata, M. O., Hannun, Y. A., \& Ng, C. K. Y. (2010). Plant sphingolipids: Decoding the enigma of the Sphinx. New Phytologist, Vol. 185, pp. 611-630.

https://doi.org/10.1111/j.1469-8137.2009.03123.x

Rennie, E. A., Ebert, B., Miles, G. P., Cahoon, R. E., Christiansen, K. M., Stonebloom, S., ... Scheller, H. V. (2014). Identification of a sphingolipid $\alpha-$ glucuronosyltransferase that is essential for pollen function in Arabidopsis. Plant Cell, 26(8), 3314-3325. https://doi.org/10.1105/tpc.114.129171 
Richter, R. P., Bérat, R., \& Brisson, A. R. (2006). Formation of Solid-Supported Lipid Bilayers $\square$ : An Integrated View. Langmuir, 22(12), 3497-3505. https://doi.org/10.1021/la052687c

Róg, T., Orłowski, A., Llorente, A., Skotland, T., Sylvänne, T., Kauhanen, D., ... Vattulainen, I. (2016). Interdigitation of long-chain sphingomyelin induces coupling of membrane leaflets in a cholesterol dependent manner. Biochimica et Biophysica Acta - Biomembranes, 1858(2), 281-288. https://doi.org/10.1016/j.bbamem.2015.12.003

Rog, T., \& Pasenkiewicz-gierula, M. (2004). Non-polar interactions between cholesterol and phospholipids $\square$ : a molecular dynamics simulation study. Biophysical Chemistry, 107, 151-164. https://doi.org/10.1016/j.bpc.2003.09.002

Róg, T., Pasenkiewicz-gierula, M., Vattulainen, I., \& Karttunen, M. (2009). Ordering effects of cholesterol and its analogues. BBA - Biomembranes, 1788(1), 97-121. https://doi.org/10.1016/j.bbamem.2008.08.022

Sampaio, J. L., Levental, I., Lingwood, D., Simons, K., Rajendran, L., Kalvodova, L., \& Kaiser, H.-J. (2009). Order of lipid phases in model and plasma membranes. Proceedings of the National Academy of Sciences, 106(39), 16645-16650. https://doi.org/10.1073/pnas.0908987106

Seelig, J. (1977). Deuterium magnetic resonance $\square$ : theory and application to lipid membranes. Quarterly Reviews of Biophysics, 3(10), 353-418.

Sibille, E., Berdeaux, O., Martine, L., Bron, A. M., Creuzot-Garcher, C. P., He, Z., ... Masson, E. A. Y. (2016). Ganglioside profiling of the human retina: Comparison with other ocular structures, brain and plasma reveals tissue specificities. PLoS ONE. https://doi.org/10.1371/journal.pone.0168794

Simenel, C., Coddeville, B., Delepierre, M., Latgé, J. P., \& Fontaine, T. (2008). Glycosylinositolphosphoceramides in Aspergillus Fumigatus. Glycobiology. https://doi.org/10.1093/glycob/cwm122

Smondyrev, A. M., \& Berkowitz, M. L. (2001). Molecular Dynamics Simulation of the Structure of Dimyristoylphosphatidylcholine Bilayers with Cholesterol, Ergosterol, and Lanosterol. Biophysical Journal, 80(4), 1649-1658. https://doi.org/10.1016/S0006-3495(01)76137-1

Sonnino, S., \& Prinetti, A. (2010). Gangliosides as regulators of cell membrane organization and functions. Advances in Experimental Medicine and Biology, 688, 165-184. https://doi.org/10.1007/978-1-4419-6741-1_12

Sperling, P., \& Heinz, E. (2003). Plant sphingolipids: Structural diversity, biosynthesis, first genes and functions. Biochimica et Biophysica Acta - Molecular and Cell Biology of Lipids. https://doi.org/10.1016/S1388-1981(03)00033-7

Tarazona, P., Feussner, K., \& Feussner, I. (2015). An enhanced plant lipidomics 
method based on multiplexed liquid chromatography-mass spectrometry reveals additional insights into cold- and drought-induced membrane remodeling. Plant Journal, 84(3), 621-633. https://doi.org/10.1111/tpj.13013

Tellier, F., Maia-Grondard, A., Schmitz-Afonso, I., \& Faure, J. D. (2014). Comparative plant sphingolipidomic reveals specific lipids in seeds and oil. Phytochemistry. https://doi.org/10.1016/j.phytochem.2014.03.023

Tjellstrom, H., Hellgren, L. I., Wieslander, A., \& Sandelius, A. S. (2010). Lipid asymmetry in plant plasma membranes: phosphate deficiency-induced phospholipid replacement is restricted to the cytosolic leaflet. The FASEB Journal. https://doi.org/10.1096/fj.09-139410

Voxeur, A., \& Fry, S. C. (2014). Glycosylinositol phosphorylceramides from Rosa cell cultures are boron-bridged in the plasma membrane and form complexes with rhamnogalacturonan II. Plant Journal. https://doi.org/10.1111/tpj.12547

Wacklin, H. P. (2010). Neutron reflection from supported lipid membranes. Current Opinion in Colloid \& Interface Science, 15(6), 445-454. https://doi.org/10.1016/j.cocis.2010.05.008

Yeagle, P. L., Martin, R. B., Lalat, A. K., Lint, H., \& Blocht, K. (1977). Differential effects of cholesterol and lanosterol. 74(11), 4924-4926.

Yetukuri, L., Ekroos, K., Vidal-Puig, A., \& Orešič, M. (2008). Informatics and computational strategies for the study of lipids. Mol. BioSyst., 4(2), 121-127. https://doi.org/10.1039/B715468B 
A

bioRxiv preprint doi: https://doi.org/10.1101/2020.10.01.313304; this version posted October 2, 2020. The copyright holder for this preprint (which was not certified by peer review) is the author/funder. All rights reserved. No reuse allowed without permission.

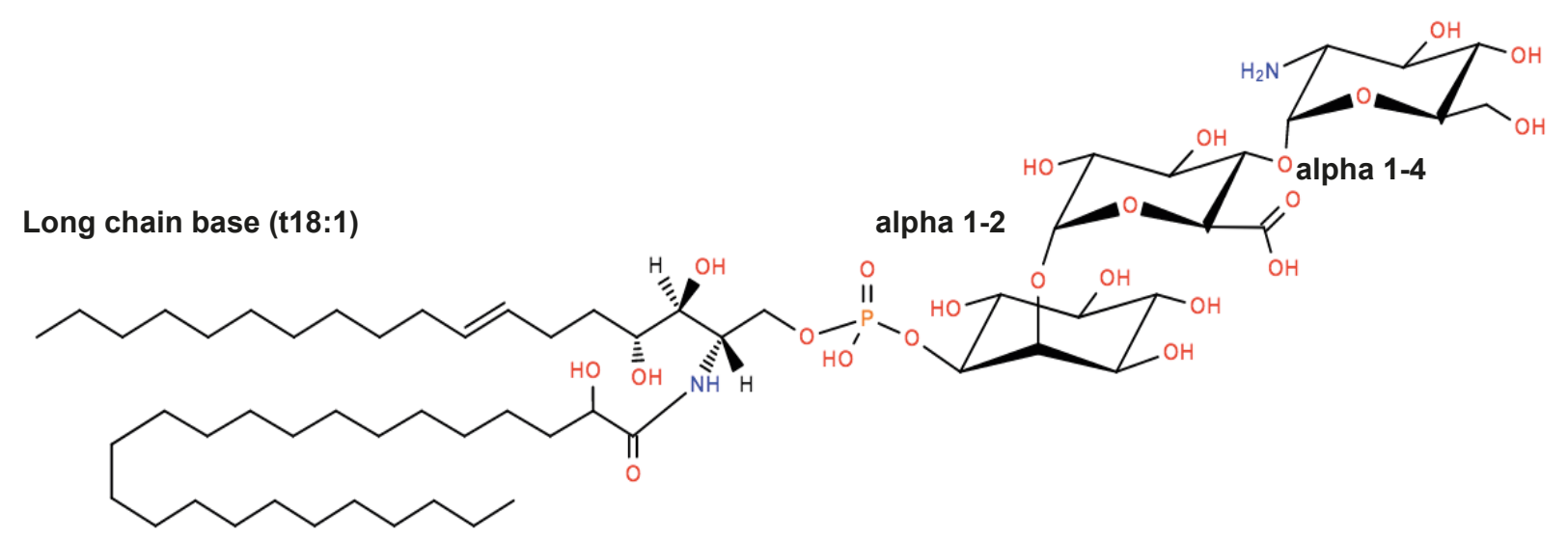

Fatty acid (h24:0)

\section{Ceramide}

Glycosyl Inositol phosphatidyl ceramide (GIPC)

B

\begin{tabular}{|c|c|c|c|c|}
\hline & & Plant Species & $\begin{array}{c}\text { Estimated GIPC by (h)VLCFA } \\
\text { content (mg/g fw) }\end{array}$ & $\begin{array}{c}\text { Main GIPC series as per } \\
\text { Cacas et al., 2016 }\end{array}$ \\
\hline \multirow{3}{*}{ Eudicots } & Plant (white parts) & $\begin{array}{c}\text { Brassica oleacera } \\
(\mathrm{n}=5)\end{array}$ & $4.3 \pm 0.9$ & A \\
\cline { 2 - 5 } & Cell culture & $\begin{array}{c}\text { Nicotiana benthamiana } \\
(\mathrm{BY}-2)(\mathrm{n}=3)\end{array}$ & $0.6 \pm 0.2$ & A B C D E F \\
\hline \multirow{2}{*}{ Monocots } & Plant (white parts) & Allium porrum $(\mathrm{n}=3)$ & $0.4 \pm 0.1$ & B \\
\cline { 2 - 5 } & Cell culture & Oryza sativa $(\mathrm{n}=3)$ & $3.4 \pm 0.2$ & B \\
\hline
\end{tabular}


Extraction process of sphingolipid

\begin{tabular}{|c|}
\hline $\begin{array}{l}\text { Raw material (non- } \\
\text { lyophilized) (1) }\end{array}$ \\
\hline$\downarrow$ \\
\hline $\begin{array}{l}\text { Grind in cold acetic acid } \\
0.1 \mathrm{~N} \text { solution }\end{array}$ \\
\hline$\nabla$ \\
\hline $\begin{array}{l}\text { Reflux in boiling }\left(65^{\circ} \mathrm{C}\right) \\
70 \% \text { ethanol } \mathrm{HCl} 0.1 \mathrm{~N} \\
\text { solution }\end{array}$ \\
\hline 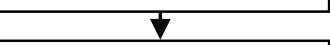 \\
\hline $\begin{array}{l}\text { Store solution at }-20^{\circ} \mathrm{C} \\
\text { for at least } 48 \mathrm{~h}\end{array}$ \\
\hline$\downarrow$ \\
\hline $\begin{array}{l}\text { Centrifuge to retrieve } \\
\text { GIPC precipitate }\end{array}$ \\
\hline 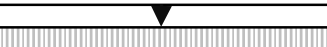 \\
\hline $\begin{array}{l}\text { Sphingolipid } \\
\text { extraction using lower } \\
\text { phase of Isopropanol/ }\end{array}$ \\
\hline $\left.\begin{array}{l}\text { Hexanel } \\
: 25 \text {, vol ratio }\end{array}\right)\left(\begin{array}{lll|}\mathrm{H}_{2} \mathrm{O} \\
: 55\end{array}\right.$ \\
\hline
\end{tabular}

\section{Purification process of GIPCs}

Sphingolipid-enriched

extract dried with silica

beads

Load silica column using chloroform

Elution of apolar lipids using $4 \mathrm{X}$ column volume of solvent mix

Chloroform/Methanol

- $4: 0(\mathrm{v} / \mathrm{v})$

- $4: 1(\mathrm{v} / \mathrm{v})$

- $2: 1(\mathrm{v} / \mathrm{v})$

- $1: 1(\mathrm{v} / \mathrm{v})$

Elution of polar lipids using a step gradient of $10 \%$ interval of solvent $A$ and solvent B mix

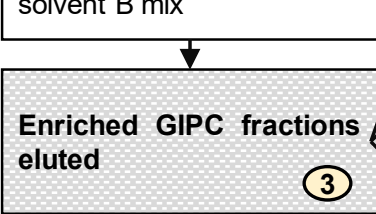

Fatty acid content by GC-MS Polar head sugar content by GC-MS HPTLC analysis

Solvent A:

Chloroform/Methanol/

$\mathrm{H}_{2} \mathrm{O}$

59: 37.5: $3.5(\mathrm{v} / \mathrm{v})$

Solvent B:

i $\mathrm{H}_{2} \mathrm{O}$

46: 42: $12(\mathrm{v} / \mathrm{v})$

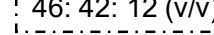

HPTLC analysis
Chloroform/Methanol/ 
bioRxiv preprint doi: https://doi.org/10.1101/2020.10.01.313304; this version posted October 2, 2020. The copyright holder for this preprint

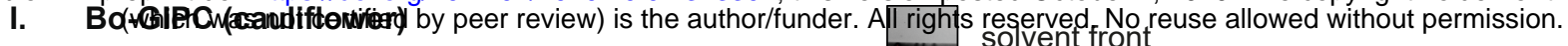

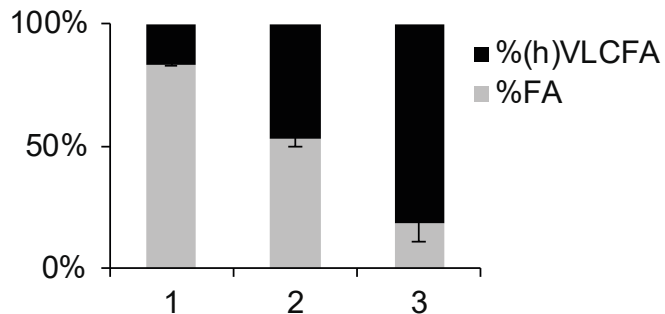

II. Nt-GIPC (BY-2)

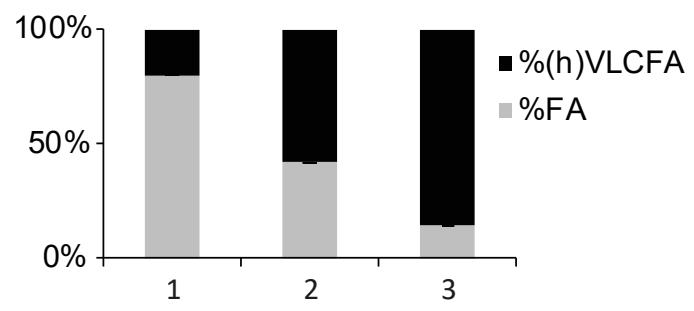

III. Ap-GIPC (leek)

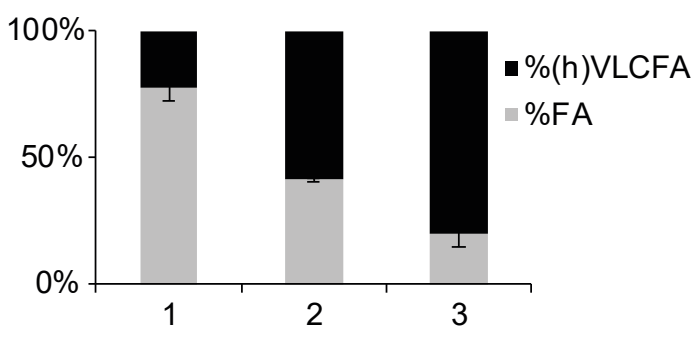

IV. Os-GIPC (rice)

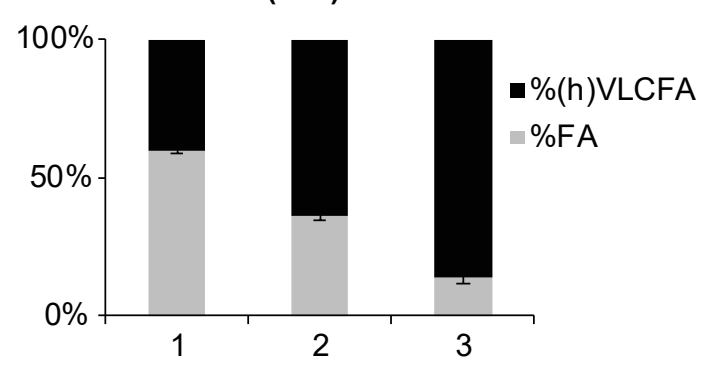

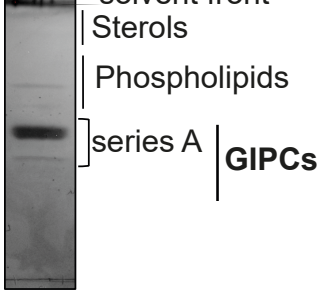
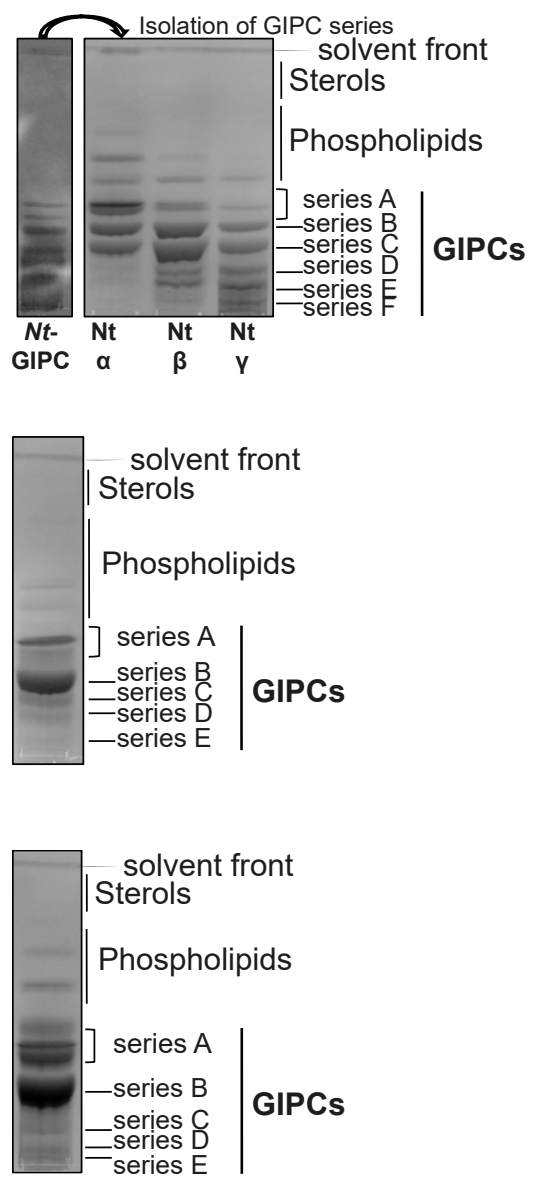


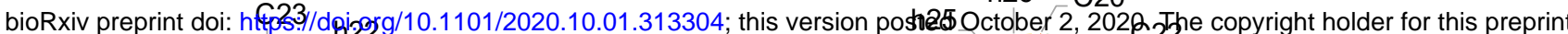
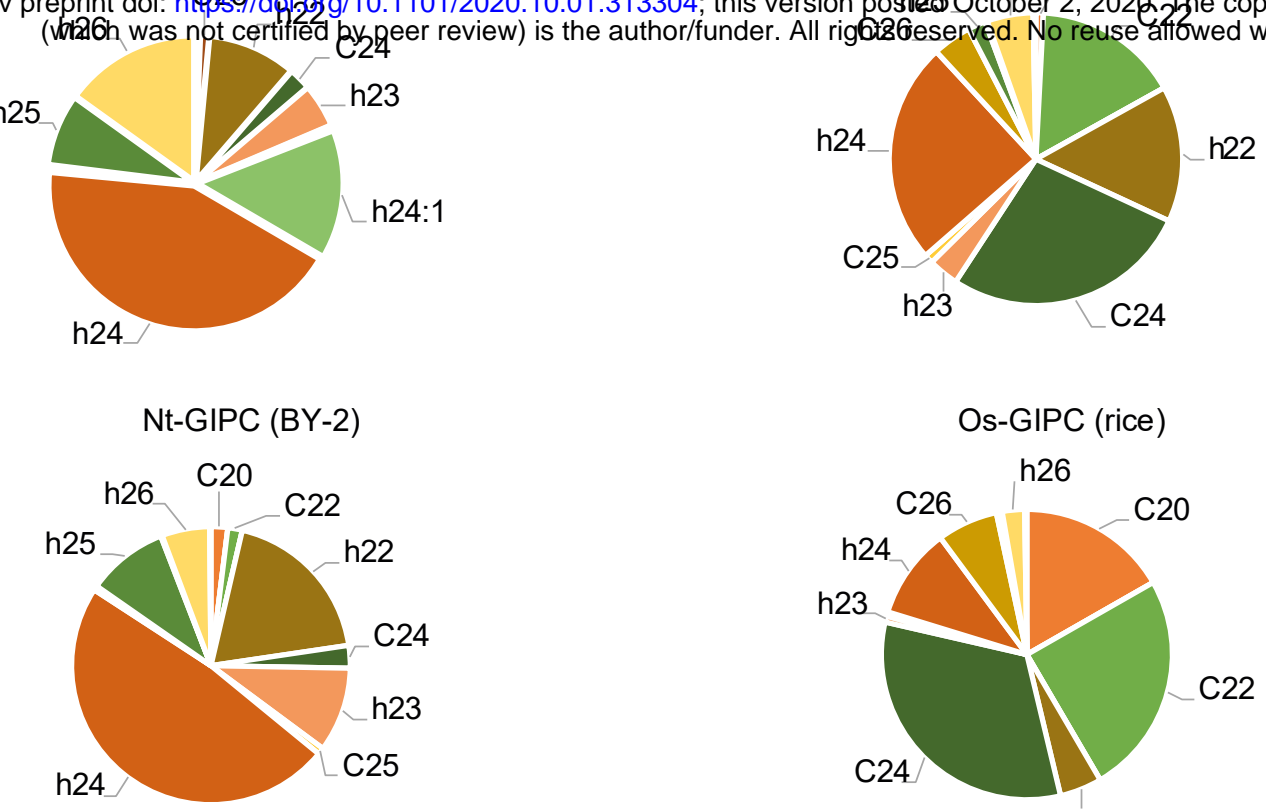

B
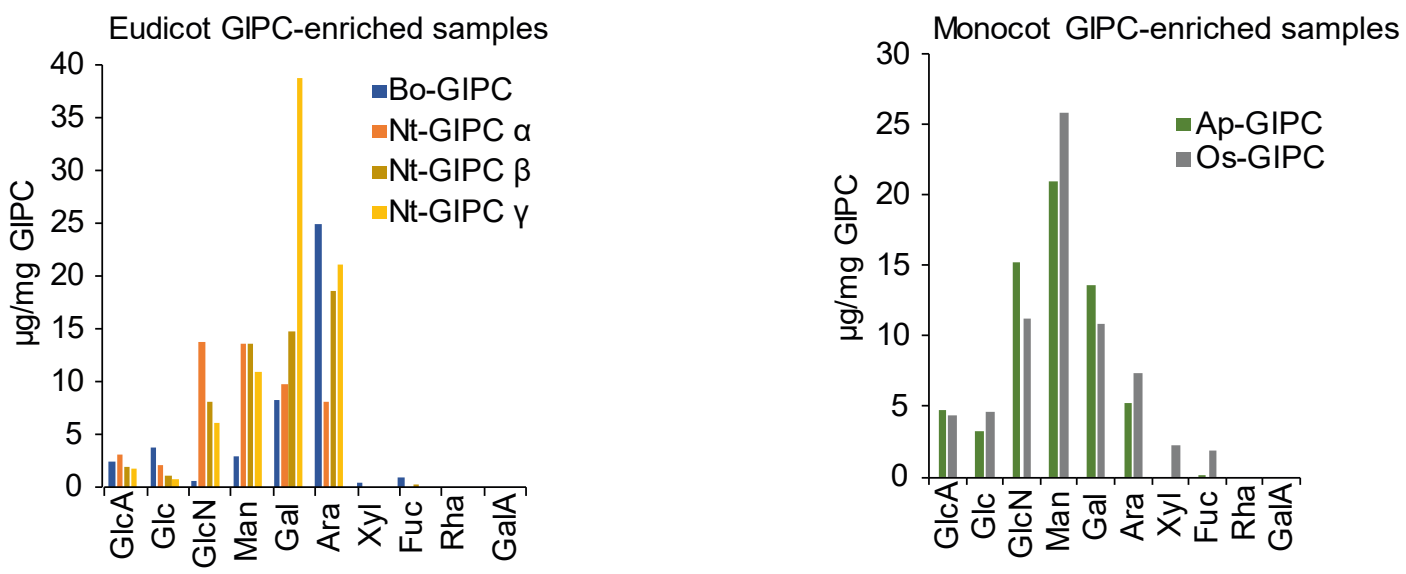

C

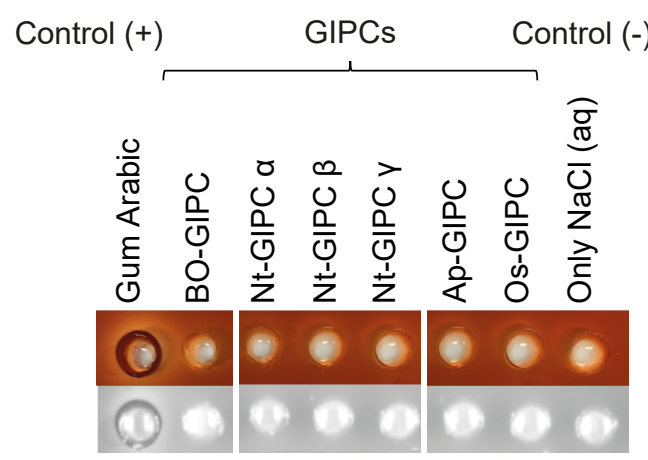


A

B

C

bio $\$$ iv preprint doi: https://doi.org/10.1101/2020.10. 313304 ; this version posted October 2, 2020. The copyright holder for this preprint

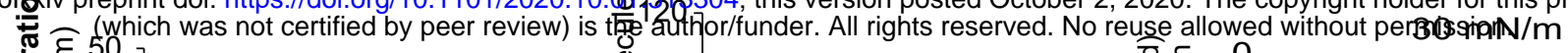
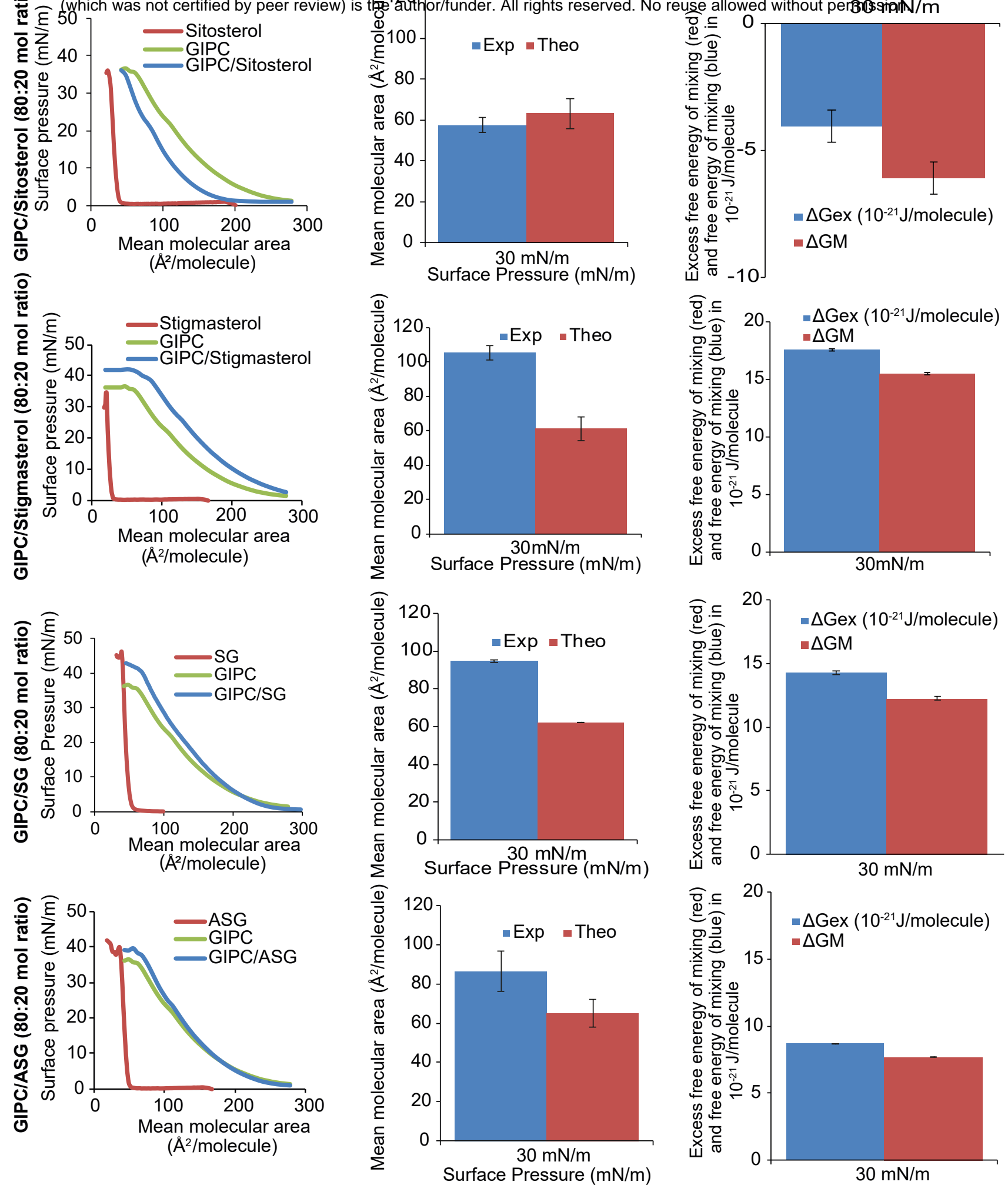
A GIPC + Sitosterol

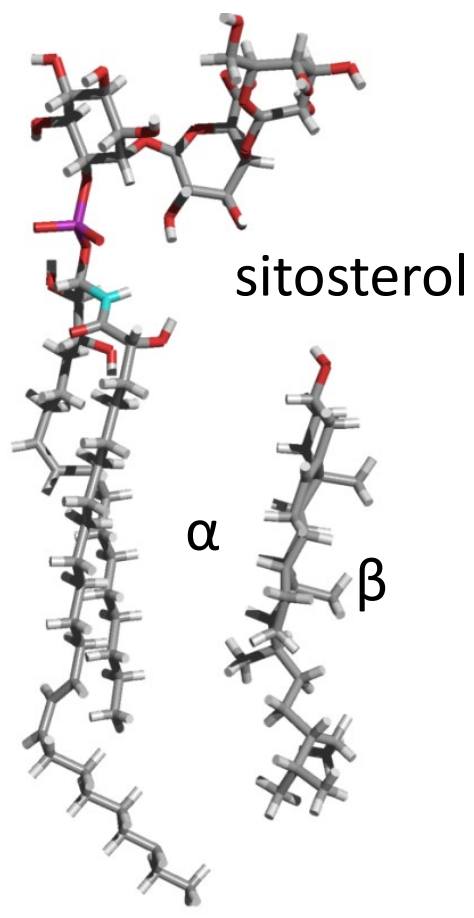

C GIPC + SG

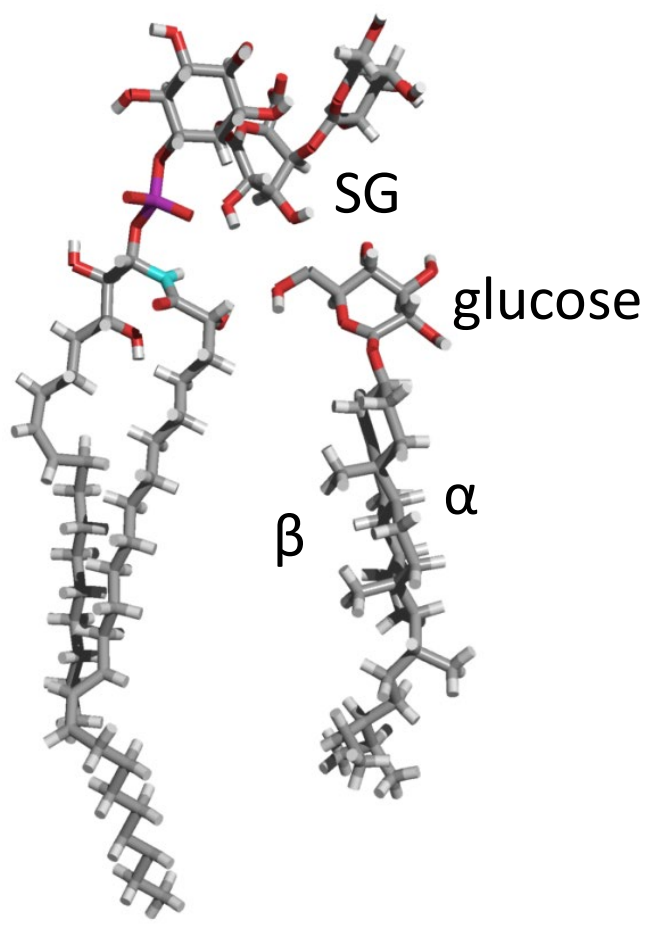

B GIPC + Stigmasterol
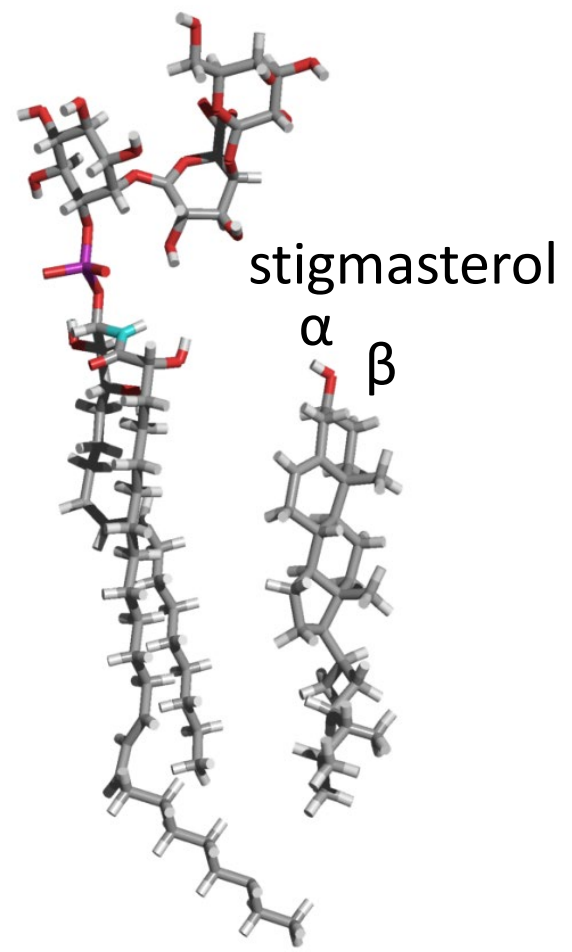

D GIPC + ASG

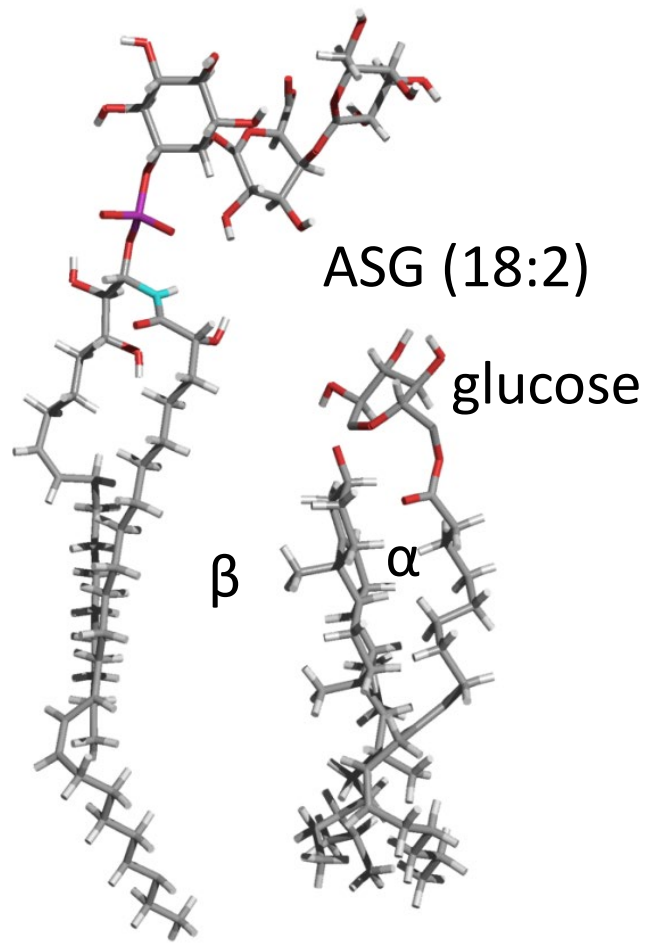


bioRxiv preprint doi: https://doi.org/10.1101/2020.10.01.313304; this version posted October 2, 2020. The copyright holder for this preprint A. Freariehthab/notethofded by peer review) is the author/funder. All rights reserved. No reuse allowed without permission.

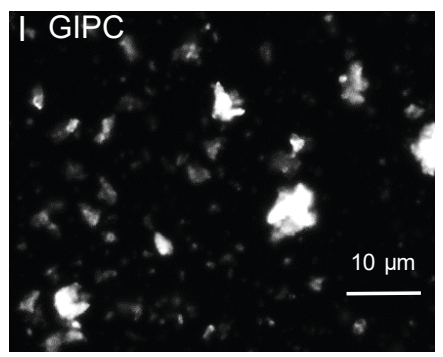

B. Teflon method

GUVs GIPC/DOPC/Sitosterol $(1 / 1 / 1$; mol ratio)

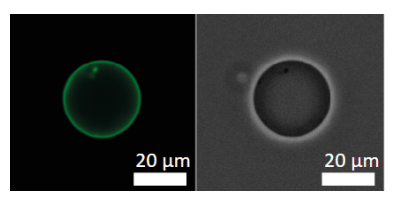

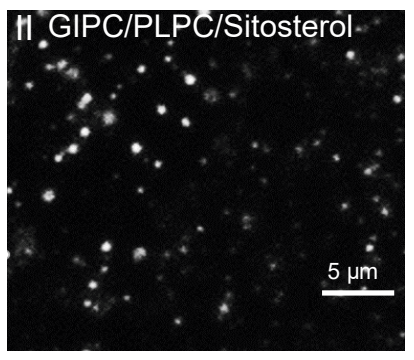

C. DLS and $\zeta$-potential (GIPC/DOPC/Sitosterol)
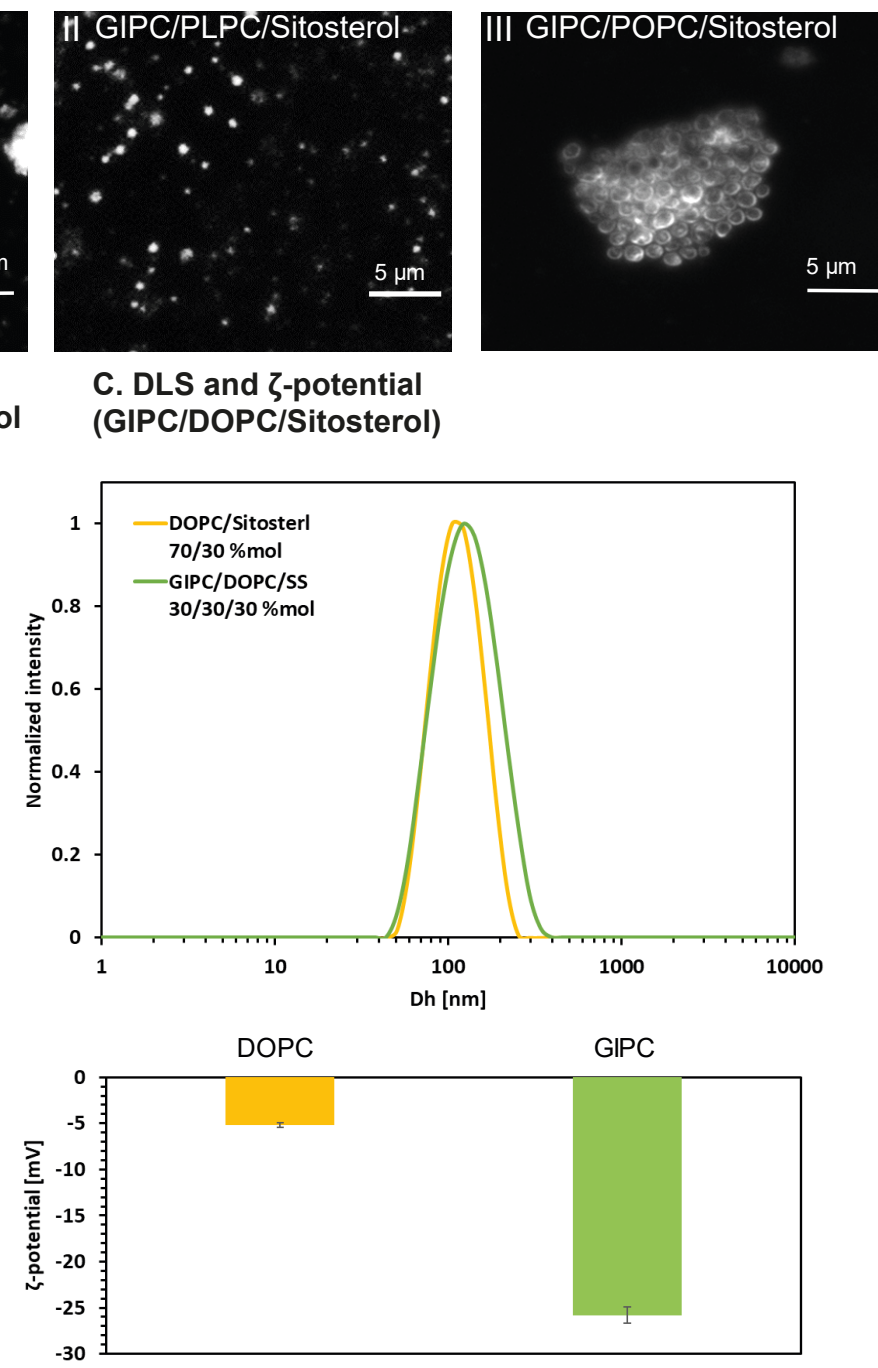
bioRxiv preprint doi: https://doi.org/10.1101/2020.10.01.313304; this version posted October 2, 2020. The copyright holder for this preprint (which was not certified by peer review) is the author/funder. All rights reserved. No reuse allowed without permission.

\section{A. Liposomes images}

i. GIPC/POPC-2H31/Sitosterol

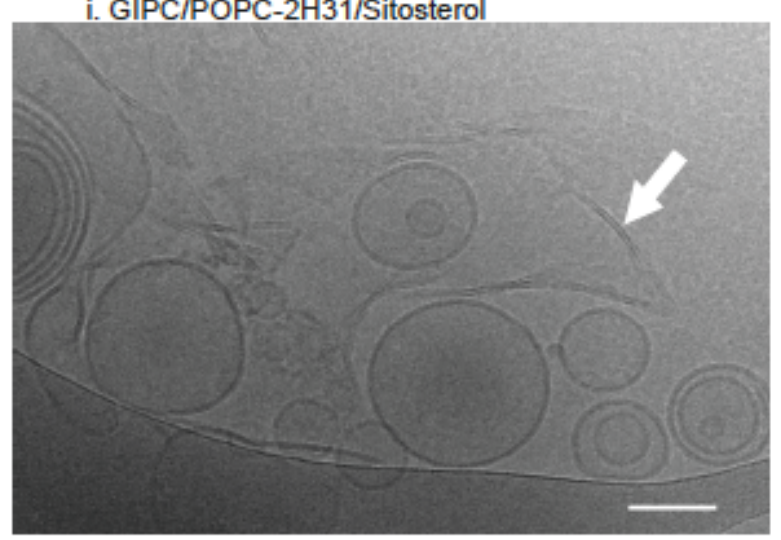

iii. GIPC/POPC-2H31/Stigmasterol ii. POPC-2H31/Sitosterol

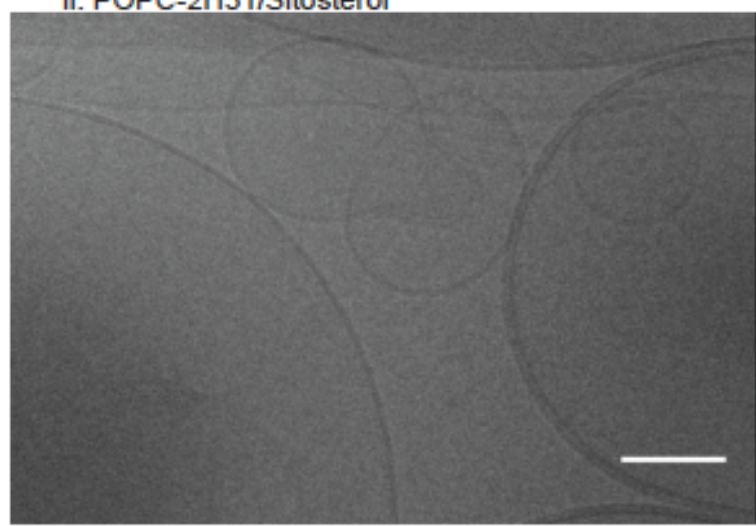

iv. POPC-2H31/Stigmasterol
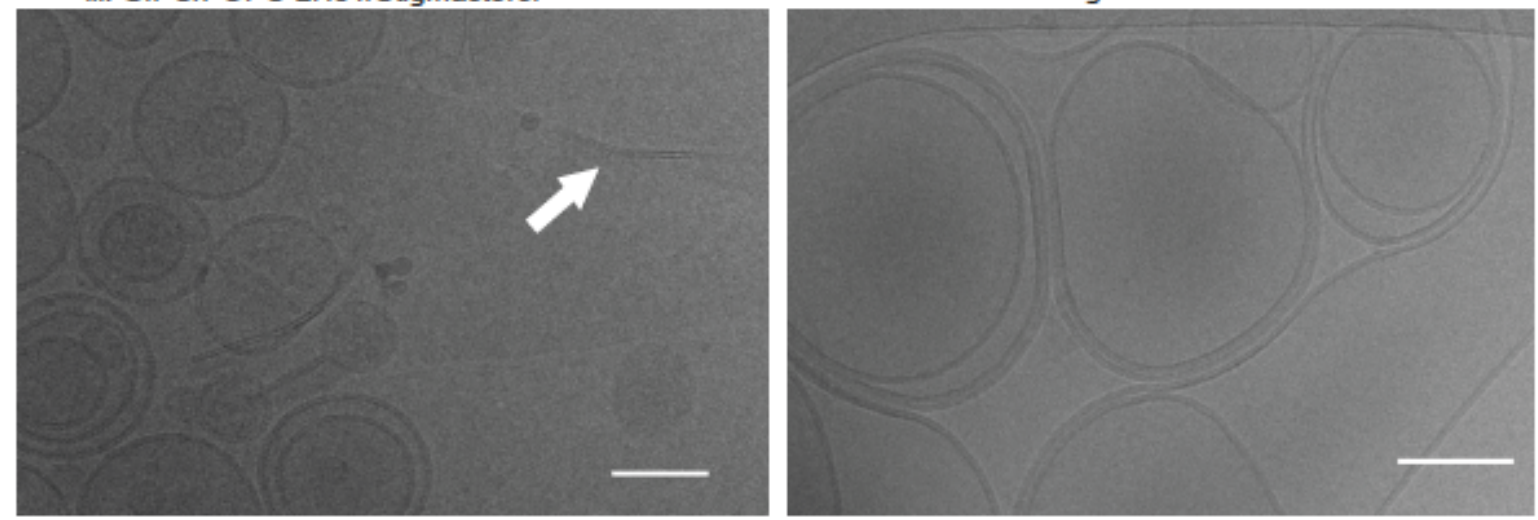

B. Liposomes Thickness (nm)

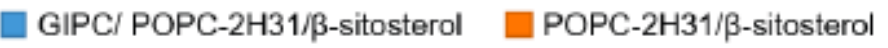 \\ GIPC/ POPC-2H31/stigmasterol — POPC-2H31/stigmasterol}

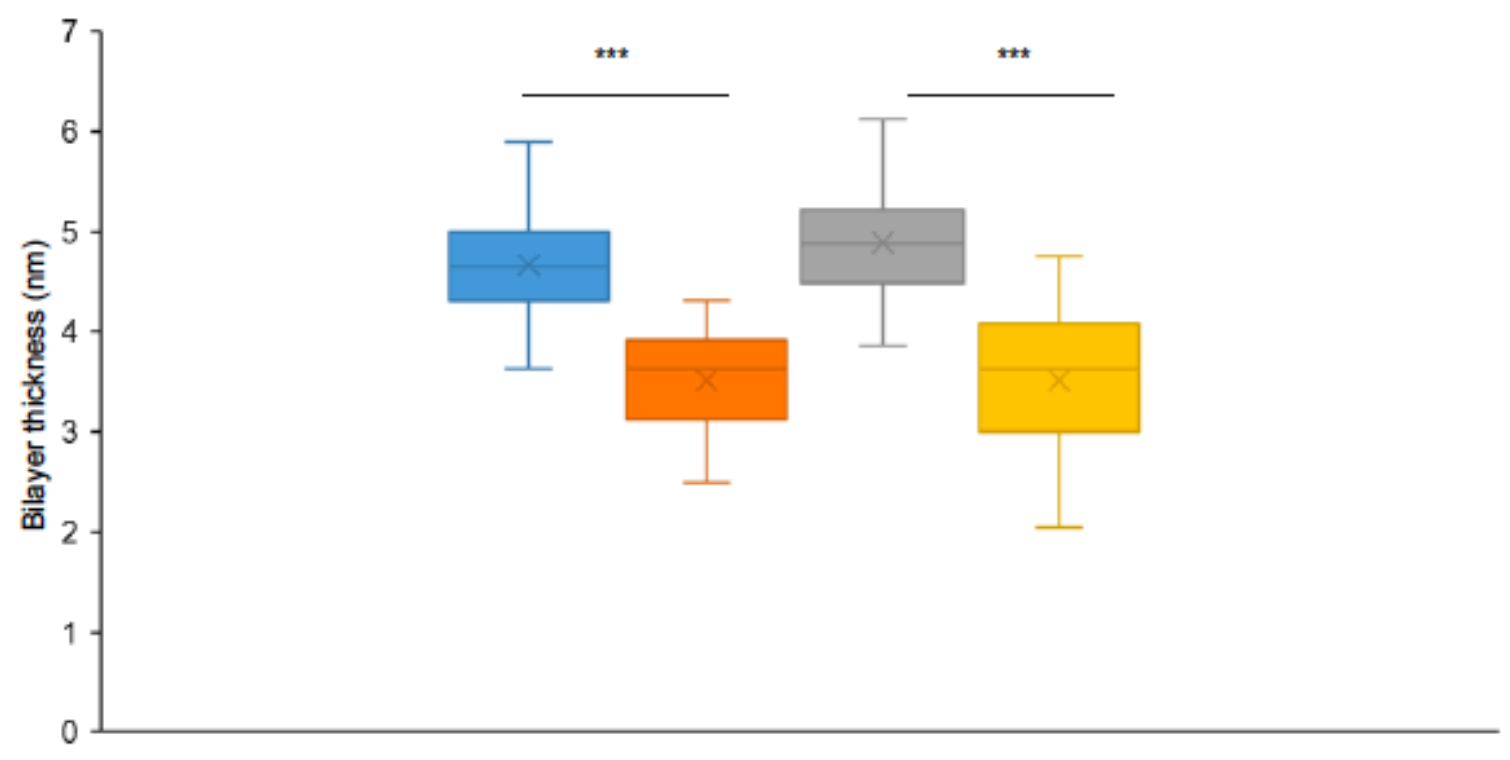


bioRxiv preprint doi: https://doi.org/10.1101/2020.10.01.313304; this version posted October 2, 2020. The copyright holder for this preprint A (which was not certified by peer review) is the author/funder. All rights reserved. No reuse allowed without permission.
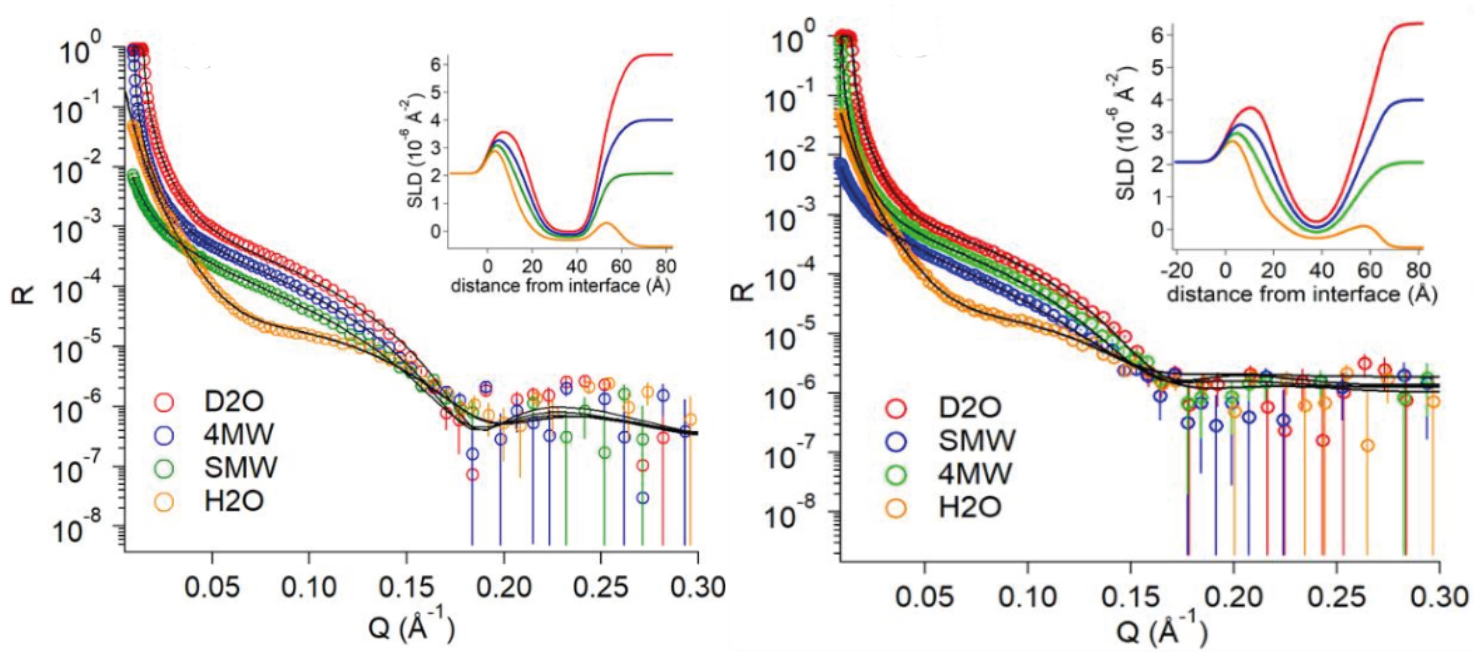

B

\begin{tabular}{|c|c|c|}
\hline Parameter & POPC/Sitosterol & GIPC/POPC/Sitosterol \\
\hline Bilayer formation & Yes & Yes \\
\hline \% solvent in the tails & 5 & 5 \\
\hline Inner Sugar $(\AA)$ & - & 4 \\
\hline Inner Head $(\AA)$ & 8 & 8 \\
\hline Tail $(\AA)$ & 31 & 31 \\
\hline Outer Head $(\AA)$ & 8 & 8 \\
\hline Outer Sugar $(\AA)$ & - & 4 \\
\hline
\end{tabular}

C

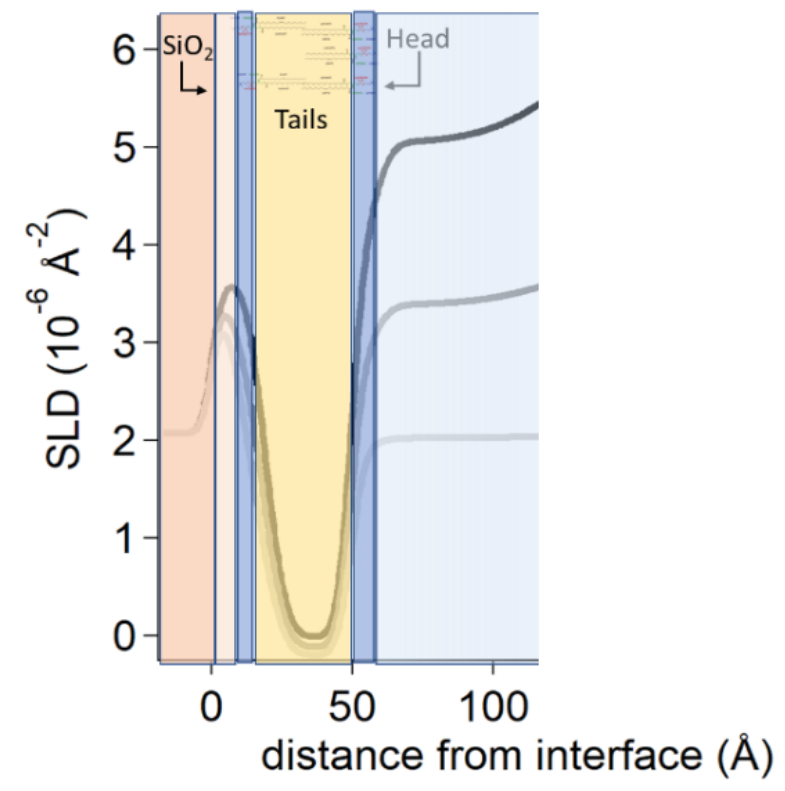


bioRxiv preprint doi: https://doi.prg/10.1101/2020.10.01.313304; this version posted Octopger 2, 2020. The copyright holder for this preprint

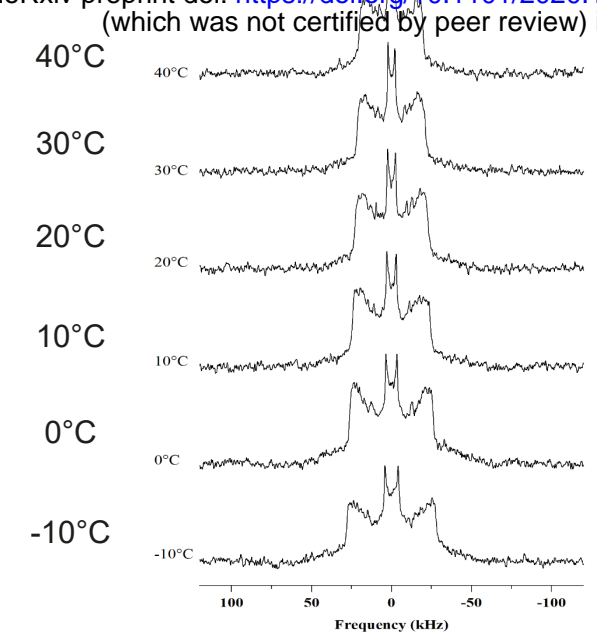

GIPC/POPCd31/sitosterol (1:1:1 mol)

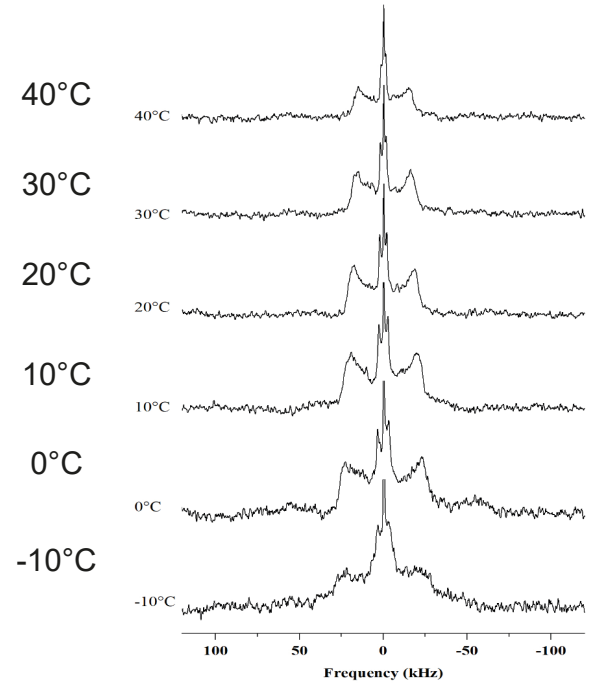

B

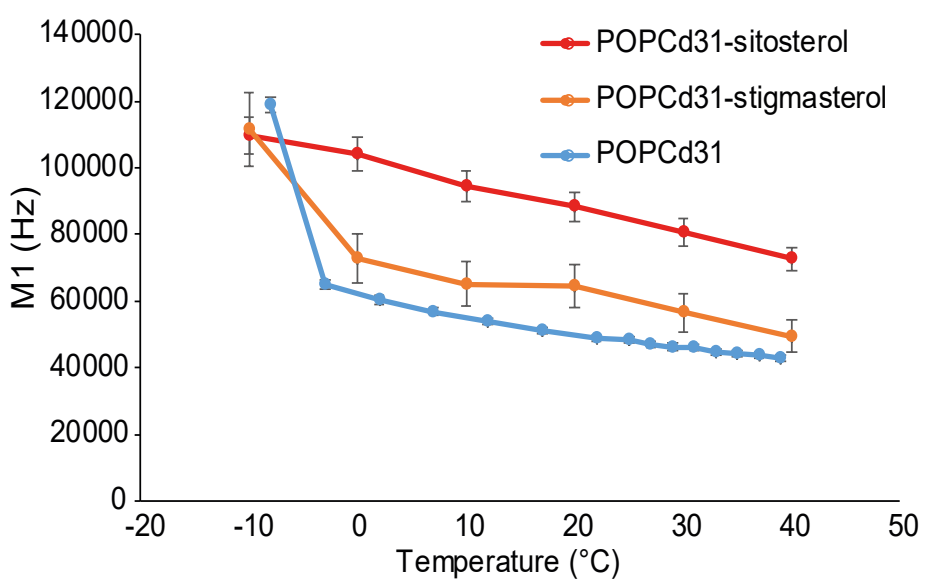

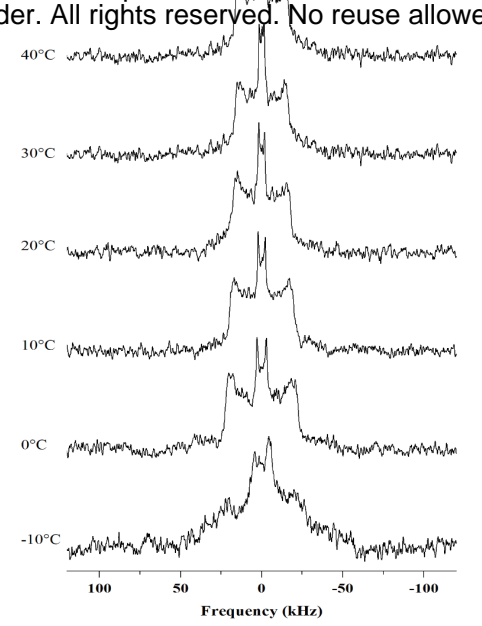

GIPC/POPCd31/stigmasterol (1:1:1 mol)

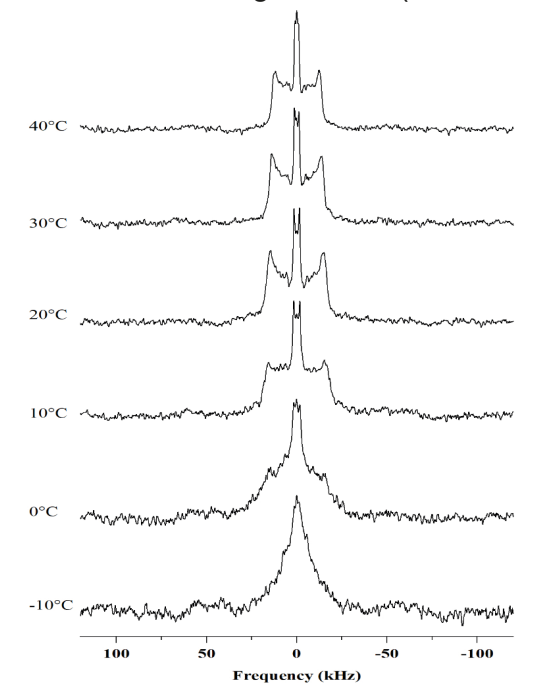

